\title{
Methodology for the assessment and refinement of friction-based dissipative devices
}

\author{
Authors: Victor Melatti ${ }^{* a)}$, Dina D’Ayala a) \\ a) Department of Civil, Environmental and Geomatic Engineering, University College London, Chadwick Building, \\ Gower Street, WC1E 6BT, UK
}

*Corresponding author. Tel.: +44 (0) 7542457437

E-mail address: ucesvme@ucl.ac.uk (V. Melatti)

\begin{abstract}
An innovative dissipative system for the seismic upgrade of historical buildings is presented. The system comprises a friction-based dissipative device able to provide energy dissipation capacity to connecting walls. To verify the compliance of the device's initial prototype with the relevant European Standards, a testing and design methodology is developed. This optimisation approach consists of: two tests loops, to identity the dependence of the dissipative device on bolt preloading, type of fasteners, frictional interfaces and environmental conditions; and a design loop where advanced 3D modelling and analysis are used to improve the design according to the experimental results. The performance is measured by variation of effective damping. This iterative procedure singles out parameters that need improvement and allows to produce two new prototypes, differing by shape and frictional interfaces. The revised prototypes prove to deliver a reliable response to cyclic loading, containing the variation of effective damping well within code requirements, even after exposure to adverse environmental conditions.
\end{abstract}

Keywords: Friction devices, historical masonry buildings, effective damping, frictional interfaces, durability.

\section{Introduction}

In the conventional strength-based design approach, seismic provisions assign a level of seismic force which defines a minimum level of lateral load capacity. The resisting system is sized to resist a combination of gravity and earthquake induced loads by adjusting the strength and the stiffness of the individual members. However, such a structure is expected to experience large inelastic deformations during the design earthquake, which in turn will determine an extensive level of residual damage in the structure and high repair costs [1]. To overcome these issues, alternative design philosophies have been developed to focus the structural design on the deformation capacity of the system rather than on its strength.

Among these alternatives, which are generally referred to as Performance-Based design solutions, is the energy-based design. In this approach, the focus is on the wave energy transferred by the seismic motion to the structure, whose effect depends on the structural properties and the characteristics of the ground motion [2]. The structural capacity is defined by the energy dissipation capacity of the resisting members, defined as the total area enclosed under the forcedeformation curves of the structure under dynamic loading. Thus, seismic design becomes the balancing of the energy input and the energy dissipation capacity of the structure.

In the last decades, strengthening solutions based on energy dissipation have been developed and implemented for engineered structures, however few applications are aimed at historic masonry buildings (HMB). For HMB, the choices of strengthening intervention are limited by conservation principles, enshrined in international and national guidelines such as the ICOMOS/ISCARSAH principles [3] and the Italian DPCM 2011 [4]. According to these documents, the benefits of a possible strengthening intervention must be weighed against the impact on the original aesthetic and structural authenticity of the building. As a result, traditional strength-only systems are still widely used to improve the seismic response of masonry structures, but often they have been proved both ineffective and incompatible with the original structures [5]. For instance, the knowledge concerning the seismic behaviour of anchors in masonry is still not exhaustive[6-9], as the experimental research is limited compared to the extended availability of literature on anchors injected in concrete elements. Therefore, it is not surprising that field observations made after recent earthquakes highlight that low masonry strength and inappropriate anchorage design (wide spacing, insufficient embedment depth) caused local and global failures of HMB retrofitted with ties [10,11].

The next section presents a review of passive control systems for HMBs. Drawing on the conclusions of this review, this paper presents an innovative passive control system specifically tailored for the seismic strengthening of HMBs. In this system, the energy dissipation capacity relays on the presence of a friction-based device which exploits and controls the rocking behaviour that monumental structures often exhibit during earthquakes [12]. The principle underlying the 
functioning of the system is that the device will provide an additional dissipative link for an existing strengthening technique, i.e. an anchoring system by means of metallic rods, which is already widely applied, standard practice for professionals in conservation engineering, cost effective and in line with current conservation recommendations. A validation programme is developed to refine the initial friction device prototype. The methodology has a generic value, as it proposes a test-analyse-redesign procedure that can be used to improve the short and long-term durability and stability of a typical friction-based device. For industrial manufacturing, the procedure is iterated until the prototype presents a stable and reliable behaviour according to the European guidelines for the validation of dissipative devices [13]. The application of the validation programme to the initial prototype of friction-based device ultimately led to the manufacturing of three alternative prototypes, of which two displayed an acceptable variation of activation load and wear of frictional material under cyclic loading conditions, while improving the general functionality, size and durability of the initial prototype.

\section{Passive control system for historical buildings}

In the balance between the seismic energy input and the energy dissipation capacity of the structure, passive control systems have the potential to play an important role even for HMB. Passive devices, such as base isolators, can modify the free vibration characteristics of the structure, shifting its natural frequencies to values characterised by modest amplification of the seismic action, thus reducing the energy demand. On the other hand, friction-based devices increase the energy-absorption capacity of the structure, by dissipating the seismic energy in the form of heat.

The review presented here comprises the energy dissipation systems applied to historical structures to improve their seismic resistance. The review is limited to passive system as it does not include monolithic art object, such as obelisks and statues, for which also active and semi-active systems have been implemented to control their rocking motion under seismic action [14]. Moreover, base isolation systems are also excluded: although they have been successfully implemented in some historic buildings [15-17], and laboratory tests [18] and numerical simulations [19] have proven the effectiveness of this technique on masonry structures, the major limitation is the topology and strength of the existing foundations. Therefore, isolation systems are seldom a viable option, without substantial geotechnical expensive work. In the case of seismic upgrade of an existing structure, the aim of including energy dissipation devices is to concentrate the dissipation in specially designed regions of the structure to minimize the inelastic behaviour of the members resisting lateral forces. In monumental buildings, the elements resisting the lateral forces are mainly walls and buttresses, when they are present. Lack of effective connections between walls and between walls and diaphragms, can lead to out of plane overturning of the perimeter walls, a recurring and highly instable failure mode. Accordingly, seismic strengthening solutions have aimed at inhibiting such a mechanism in favour of higher stiffness and strength of the structure. For the St Giovanni Battista Church in Carife (IT), Shock Transmitter Units (STU) were used to connect the masonry walls to a new steel roof structure [20]. These hydraulic devices allow the connected elements to move relatively to each other under low-velocity actions, such as thermal variations. On the other hand, under high velocity actions, such as earthquakes, the viscosity of the fluid responds with high stiffness: the roof structure then behaves like a rigid diaphragm redistributing the shear inertia forces among all loadbearing walls.

In contrast, in the last decade post-earthquake surveys showed that several churches survived the earthquake practically undamaged despite the activation of an out-of-plane mechanism of the perimeter walls. Experimental tests confirmed that rocking motion without overturning allows a wall to sustain a range of accelerations that exceed the limit computed with the simplified "quasi-static" assumption [21]. Therefore, use of rocking motion of walls to dissipate seismic input energy while controlling the rocking amplitude to ensure safety, is the strategy employed for the structural restoration of the Basilica of S. Francesco at Assisi, whereby STU were used in combination with Shape Memory Alloy Devices (SMADs) [22]. Owing to their particular stress-strain relationship, the SMADs can allow "controlled displacements" of the anchorage, lessening the stiffness of the connection and thus preventing the typical pull-out failure of traditional steel-ties anchorages. Shake table tests confirmed that the addition of a ductile link as a SMAD could achieve an acceleration reduction up to $60 \%$ at the connection level compared to traditional anchorages, such as horizontal steel ties.

On a similar concept, [23] developed a dissipative device based on the plastic yielding of steel. The proposed dissipative anchoring system comprises a device designed to have variable cross-sectional area and lower yielding strength than the ties is connected to. This means that, for a given level of axial force acting on the assembly, different sections feature different stress levels, but only the dissipative element undergoes yielding, as proven by extensive laboratory activities. The device underwent on site validation by installation in the Oratory of S. Giuseppe dei Minimi in L'Aquila, subjected to structural damage during the seismic events of April 2009. The system was able to deliver a performance similar to that of the standard anchors, but with the non-negligible advantage of preventing damage at the anchor heads. Compared 
to SMADs a steel-yielding device has the advantage of being less expensive and easier to design given the constitutive law of steel is well known by professionals.

The DIS-CAM (DISsipative Active Confinement of Masonry) system is also based on the post-elastic behaviour of steel. This was proposed by [24] for the seismic upgrading of the drum supporting the dome of the S. Nicolò's church in Catania (Italy). The system consists of angle steel plates welded in correspondence to the corner of the openings, connected by net of steel ribbons. During a seismic event, the dissipative capacity is provided by rocking behaviour of the masonry panels, which stress the steel elements over their elastic limit. A net of steel ribbons limits the allowable rocking motion of the panels to prevent the ultimate failure of the structure. The system was applied on scaled models representing the shape and materials of the real dome and experimentally tested by shaking table for increasing intensity of natural earthquake records. Compared to other strength-only solutions, the dissipating capacity of the dissipative elements allowed the dome to resist higher seismic accelerations without damage.

Friction-based dampers have been successfully employed for the past two decades [25] as component of passive control systems for new or existing structures. The largest number of applications of friction dampers are for bracing systems, such as the Pall Friction Dampers [26], and beam-to-column connections in steel and reinforced concrete structures, which were firstly developed by [27]. In general, the reason for preferring friction-based dampers to other methods is that they provide greater energy dissipation for the same amplitude and load peak, while being economical to produce and requiring relatively low maintenance [28]. Moreover, friction dampers have a rigid-plastic behaviour, which normally depends only on two parameters, namely the clamping force and the friction coefficient of the interfaces in contact. Despite the abundance of friction-based devices available for RC and steel buildings, very few friction dampers have been designed and tested for historic masonry structures.

An energy dissipation device based on friction was developed by [29] in 2015, but it is limited to mortar-less walls made of semi-interlocking masonry (SIM). Compared to traditional masonry walls, SIM infill panels act as energy dissipation devices, as they dissipate earthquake energy through friction between bricks, while the out-of-plane relative movement is hindered. This system proves that friction-based systems significantly improve the energy dissipation capacity, but the SIM dissipative device is not suitable for historical buildings, where the mortar is typically the bonding agent between blocks and the relative motion between courses would represent a source for local and global structural failure. A solution for single nave churches was proposed by [30], where energy dissipation is obtained by installing frictionbased devices at the corners of the roof diaphragm, enabling the deformation of the structure under lateral loads. Tests on a full-scale $2 \times 2 \mathrm{~m}$ portion of such "dissipative diaphragm" shows that the use of friction dampers reduced the shear demand transferred to the facade in comparison to a stiffer roof. The average maximum drift demand for such flexible diaphragm, $0.6 \%$ as reported by the authors, is well within the range of the undamaged transversal response of masonry arches, measured in other experimental campaigns [31,32]. Nonetheless, for churches with vaulted ceilings or in presence of precious finishing such as frescos and bas-reliefs, such maximum drift may be unacceptable: stronger and/or stiffer roof diaphragm would be required to limit the maximum drift within acceptable values, which from experimental tests can be set at about $0.15-0.2 \%$ [30]. Nonetheless, increasing the stiffness of such "dissipative diaphragm" is not recommended, as it would inhibit the self-centring capabilities of the system, leaving remarkable residual deformations. A friction-based device, developed and patented by D'Ayala et al. [33] is shown in Fig. 1a. The device, designed to increase the performance of grouted tie anchors in the non-linear range, is connected to stainless-steel profiles (Fig. 1b) that are encased in a fabric sleeve filled with grout. The assembly is then installed in holes core-drilled in the structure to connect orthogonal walls or walls to stiff diaphragms (Fig. 1c). When the grout is injected in the flexible sleeves, they expand, thus moulding into the shape and space within the wall, providing mechanical and chemical bonding.

a)

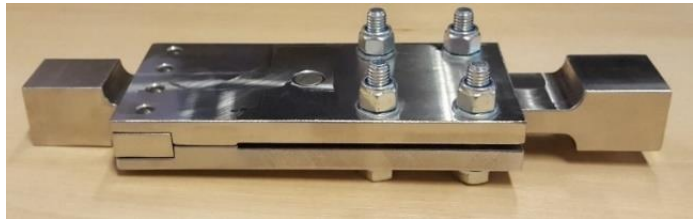

b)

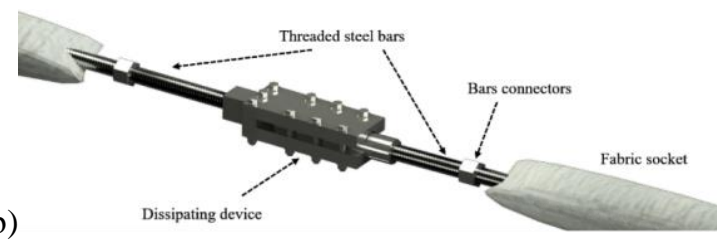

c)

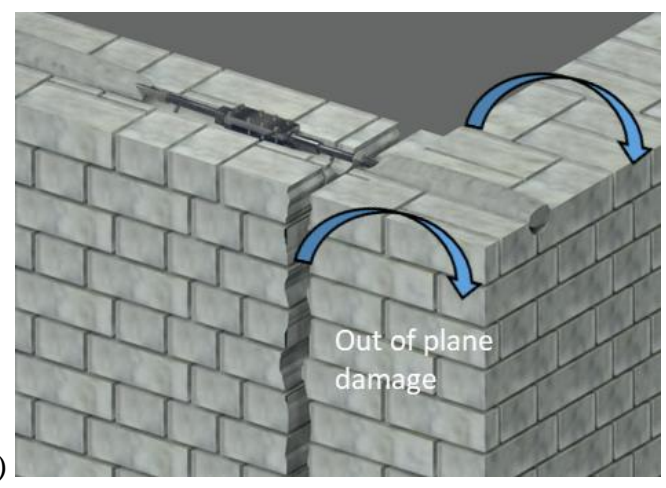

Fig. 1: a) Patented design of the dissipative device, b) Insertion of the device at the connection between perpendicular walls 
The dissipative anchoring system is schematically represented in Fig. 2a by a system of springs representing the grouted anchors and the device. The strength of the grouted ties, $F_{b o n d, s i d e}$ and $F_{b o n d, f r o n t}$, is determined once the shear strength developed between the ties and the parent material, and the embedment length $L_{t}$ are known. Conversely, the device's strength $F_{\text {friction,dev }}$ is equal to the friction resistance the device is designed to provide. The system is adjusted to have $F_{\text {friction,dev }}$ at the bottom of the anchor strength hierarchy, so that, during an earthquake (Fig. 2b), the detachment of the façade from the side walls triggers the activation of the device and prevents the bonding failure of the anchors. Therefore, the device is tuned to activate for a slippage load equal to the estimated bonding resistance of the portion of wall which the anchors controls, and smaller than the bond that the two grouted steel elements can provide. With reference to Fig. $2 b$, the design check is expressed by Eq. 1 :

$$
F_{\text {friction,dev }}<\min \left(F_{\text {bond,side }}, F_{\text {bond,front }}\right)
$$

The sliding motion is bounded to a maximum displacement by a fix pin, with strength capacity greater to the anchor bond strength, and results in the dissipation of the input seismic energy.

a)

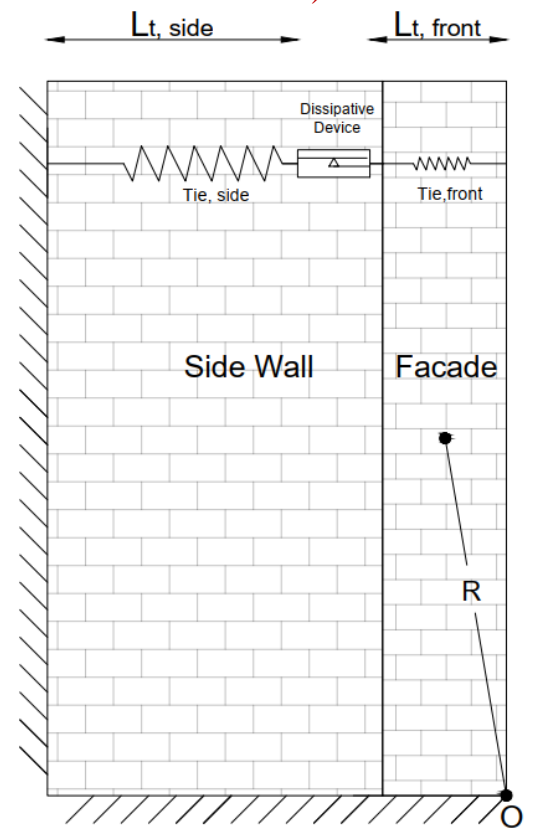

b)

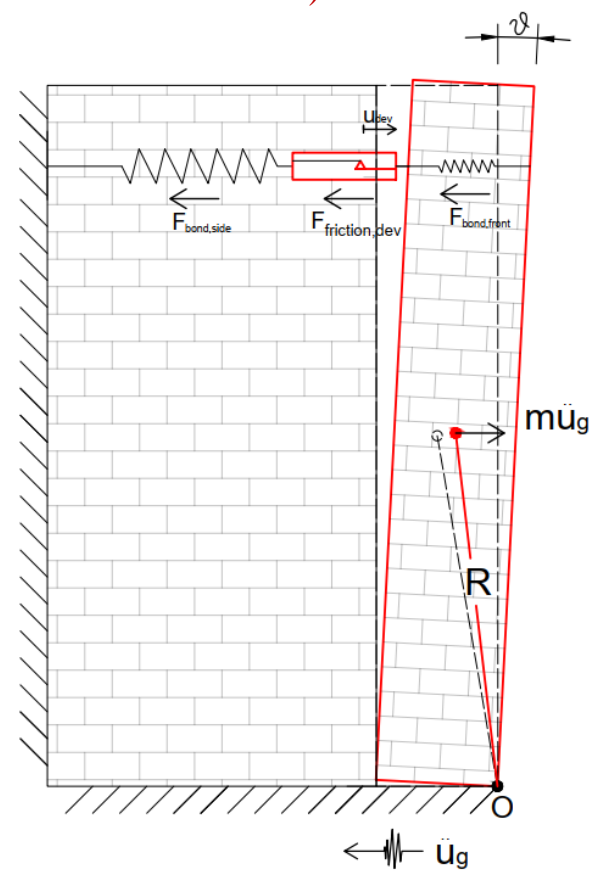

Fig. 2: a) Corner connection strengthened by dissipative anchoring system [33]: a) resting position of the wall, b) tilted configuration of the wall due to base acceleration and activation of the device.

The dissipative anchoring system can be installed along the wall height, so that the energy dissipation can take place even for small rotations of the rocking motion. It is suitable both for connections that have already experienced a crack as a result of a seismic event or for undamaged connection. In the latter case, a structural analysis is required to determine the optimal location where the devices should be placed, namely where a vertical/diagonal crack is likely to happen. The friction device prototype showed good results in terms of energy dissipation capacity and versatility. Under sinusoidal cycles of loads, the device showed repeatable performance, independent of amplitude and frequency of imposed load as well as of temperature. Moreover, the design ensures that the installation of the system has a minimal impact on the building in terms of loss of original material and aesthetic compatibility. Nonetheless, the performance of the device was not consistent throughout the experimental campaign, limiting its practical application [34]. The promising results of the patented friction-based device proposed in [33] led to the research reported in this study, which aims at identifying the causes of the abovementioned inconsistency and refine the prototype's geometry to achieve a stable and reliable behaviour. The iterative methodology followed during this investigation is laid out in the next paragraph. 


\section{Methodology for the Assessment and refinement of friction-based dissipative devices}

Limited application of dissipative devices to HMB, as seen in the previous section, is mainly due to lack of code's guidance on their design, elevated costs of the system's materials, and the limited knowledge and difficulty of modelling their behaviour. The dissipative anchoring system presented herein aim at tackling these issues, as it is designed to be readily available, affordable, and straightforward to control and design.

This device falls into the category of slotted bolted connections (SBCs) [25]: a set of bolts that pass through the assembly are tightened to compress the pair of outer plates around the sliding element, thus generating a resisting force to friction, and dissipate energy when it is activated. The design of a friction-based device is based on the Coulomb equation of friction:

$$
F_{\text {fric }}=n \mu F_{\text {perp }}
$$

which states that the maximum friction force $F_{\text {fric }}$ generated by the slippage of elements in contact is dependent on the applied perpendicular force $F_{\text {perp }}$, the friction coefficient, $\mu$, and the number of surfaces in contact, $n$. Any force smaller than $F_{\text {fric }}$ is not able to cause the relative motion between the elements.

For friction devices composed by three superposed plates, $n$ is generally assumed equal to two. A central pin is designed to ensure that the sliding motion remains within the designed allowable range. Given the material of the contact interfaces and the squared cross-section of the internal sliding element, this prototype is herein referred as SteSq prototype.

Following extensive testing, the performance of the SteSq prototype is considered unsatisfactory as the resistance to motion increases with the number of cycles [34]. Therefore, an iterative strategy, reported in Fig. 3, is developed to investigate the stability and durability of the device over a short-term and a long-term assessment framework, the results of which shall inform the design, refinement and production of new prototypes.

The first task (Short-term Assessment) explores the stability of the device's performance and changes in frictional properties of parts in contact due to progressive wear.

In general, wear of sliding surfaces can be caused by many mechanisms such as adhesion, abrasion, oxidation, delamination, melting, and more [35]. It follows that the wear rate $S w$ is a function of many factors: the normal force, $F_{\text {perp }}$ acting across the sliding surfaces, their relative velocity, $v$, their initial temperature, $T_{0}$, and the thermal, mechanical and chemical properties of the materials which meet at the surface. For each of the mechanisms, $S w_{\mathrm{i}}$ can be defined as:

$$
S w_{i}=f\left\{F_{\text {perp }}, v, T_{0}, \text { th. }, \text { mech., ch. properties }\right\}
$$

Among these, the one affecting the SteSq prototype is mainly the effect of plasticity-dominated wear, consisting in the removal of slivers of metal from one or both surfaces [36]. If two plates of the same material are in contact, as in the case of steel on steel plates, severe wear with high wear rate is often registered: this process makes the contact surfaces rougher than the original state and a hardening behaviour of the friction coefficient is recorded. In contrast, if two different materials are experiencing sliding in dry friction conditions, mild wear with a low wear rate will be recorded on the surface with lower elastic modulus $E$. The removal of material from contact surfaces results in thinner plates and a reduction of the clamping force, determining a softening behaviour of the slippage load. In both cases, the hysteretic behaviour of the damper is greatly affected by the wear and extensive laboratory tests need to be performed to design solutions able to minimize this material loss during dynamic loading.

In the proposed methodology, two activities are set out for the assessment of the short-term behaviour of the device: first, the empirical correlation between the applied tightening torque $M$, and the tension transferred to the bolts $F_{p c}$ is determined. A parameter $k_{m}$ is computed to investigate the influence of the device's geometry and the type of nut on the bolts tightening load. Secondly, the variability of the dissipative capacity of the device under external cyclic loading for different tightening load is investigated. The values of slippage force is recorded for each cycle, and the effective viscous damping $\xi_{e f f b}$ is computed according to EN 15129 [13] provisions. The amplitude of the variation of $\xi_{e f f b}$ throughout the test determines the stability of the device.

According to the EN 15129's requirements [13] for displacement-dependent devices, devices shall undergo 20 cycle reversals with a variation of the effective viscous damping $\Delta \xi_{e f f b}$ not greater than $\pm 10 \%$ of the value recorded at the third cycle. At the end of each testing session, a visual inspection of the device determines the wear rate $S_{w}$ due to the friction of components in contact. Depending on the friction materials involved, the factors of Eq. 2 that affect $S_{w}$ are experimentally determined. 
If the parameter $\Delta \xi_{e f f b}$ is above the target tolerance of variation, the prototype should be redesigned exploring different solutions (change in device's shape, number/size of bolts) and interface materials. This task is referred as "design refinement" in the proposed methodology, and it is carried out "virtually" by testing the solutions through a detailed finite element model, which also provides insight in the stress distribution within the device. The optimised prototypes are manufactured according to the design modifications that have the potential of improving the device's behaviour and tested again for short-term stability.

Finally, the prototypes that satisfy the requirements for the short-term assessment undergo a process of durability characterization. (see Fig. 3, long term assessment box).

A device might be non-operational for years before an earthquake strikes and its expected response might be affected by several factors. For bolted slotted devices, the loss of initial bolt preload is the main cause of instability over time. Immediately after tightening, the bolt experiences an initial loss which is mainly associated with the installation process, and increases with the magnitude of the applied preload, especially if the bolt is tightened beyond its yield limit [37]. Beyond this, a mid-term loss occurs continuously during its operational life, the loss-rate becoming stable over time. [38] identified a direct correlation between the loss of preloading and the service loading conditions highlighting that the use of a conical disk springs reduces the loss of preload over time. High temperature may also influence the bolt preload. Test conducted on bolts grade 8.8 showed that temperatures above $300^{\circ} \mathrm{C}$ activate creep and relaxation phenomena reducing the yield strength of the steel, which produces a proportional load relaxation in the fastener [39]. Nonetheless the service operability of dissipative devices never reaches such range of temperatures in typical civil applications, as studies on frictional dampers have confirmed [40].

Another source of possible long-term instability for friction-based dissipative devices is the oxidation of the elements composing the device due to environmental conditions. Stainless steel, an alloy of iron, chrome and carbon, is typically used as principal material in such devices, as it presents good mechanical strength, and better resistance to environmental corrosion than iron steel [41]. The chromium ions react with oxygen forming a protective stable film which acts as a barrier for additional corrosion. Nonetheless, friction devices might include other metals in combinations with stainless steel. For instance, brass and aluminium are typically used as friction shims to improve the stability of the hysteresis loops, while different steel alloys might be used for the fasteners to achieve higher strength. In certain circumstances, such mixed-material design can lead to galvanic corrosion in one of the partner materials. This phenomenon takes place when two metals in contact have different corrosion potential in presence of an electrolyte. Contrary to widespread belief, the difference in potential in an electrochemical cell alone is not a good indicator of the actual risk of galvanic corrosion [41]. The decisive factor is the difference in potential observed under real operating conditions. As the proposed device is intended to be inserted within the thickness of historical masonry walls, the corrosion process can be simulated by exposing the prototype to the environmental conditions typically observed in the masonry fabric of historical walls. As reported by $[42,43]$, monitoring the relative humidity $(\mathrm{RH})$ within the wall thickness, typical ranges are between $65 \%$ and $70 \%$ when the outside temperature and $\mathrm{RH}$ range between $10^{\circ} \mathrm{C}$ and $20^{\circ} \mathrm{C}$ and $40 \%$ to $90 \%$ respectively. For outdoor temperature between $0^{\circ} \mathrm{C}$ and $10^{\circ} \mathrm{C}$, the in-wall $\mathrm{RH}$ reaches values of about $85-95 \%$, meaning that the walls are subject to prolonged saturation in vapor phase and therefore are highly likely to contain liquid water. These conditions might trigger galvanic corrosion in the anodal element. Moreover, rainwater has a specific conductivity three orders of magnitude greater than pure water, causing an acceleration of the oxidation process. For these reasons, the risk of galvanic corrosion is explored in the long-term durability task. The devices are exposed to temperature and humidity cycles that simulate the environmental conditions of a real-case installation to explore the resistance of the prototypes to corrosion. After conditioning, the devices are tested with the same protocol used in the "short-term assessment" to determine any loss in performance stability due to environmental exposure. The maximum variation of effective damping after the aging process (referred to as $\Delta \xi_{\text {effb_aged }}$ ) shall be within $\pm 15 \%$ as indicated by [13].

The application of the testing methodology to the SteSq device is discussed in Section 4, the design refinement in section 5 , the short-term assessment of the revised prototypes in section 6 and the environmental conditioning and testing of the new prototypes for long-term assessment are presented in section 7. The results of the experimental and numerical activities show that the revised prototypes deliver a more stable behaviour under dynamic loading and show a better resistance to corrosion over time. These results highlight that the proposed methodology can be applied for the assessment and refinement of friction-based dissipative devices. 


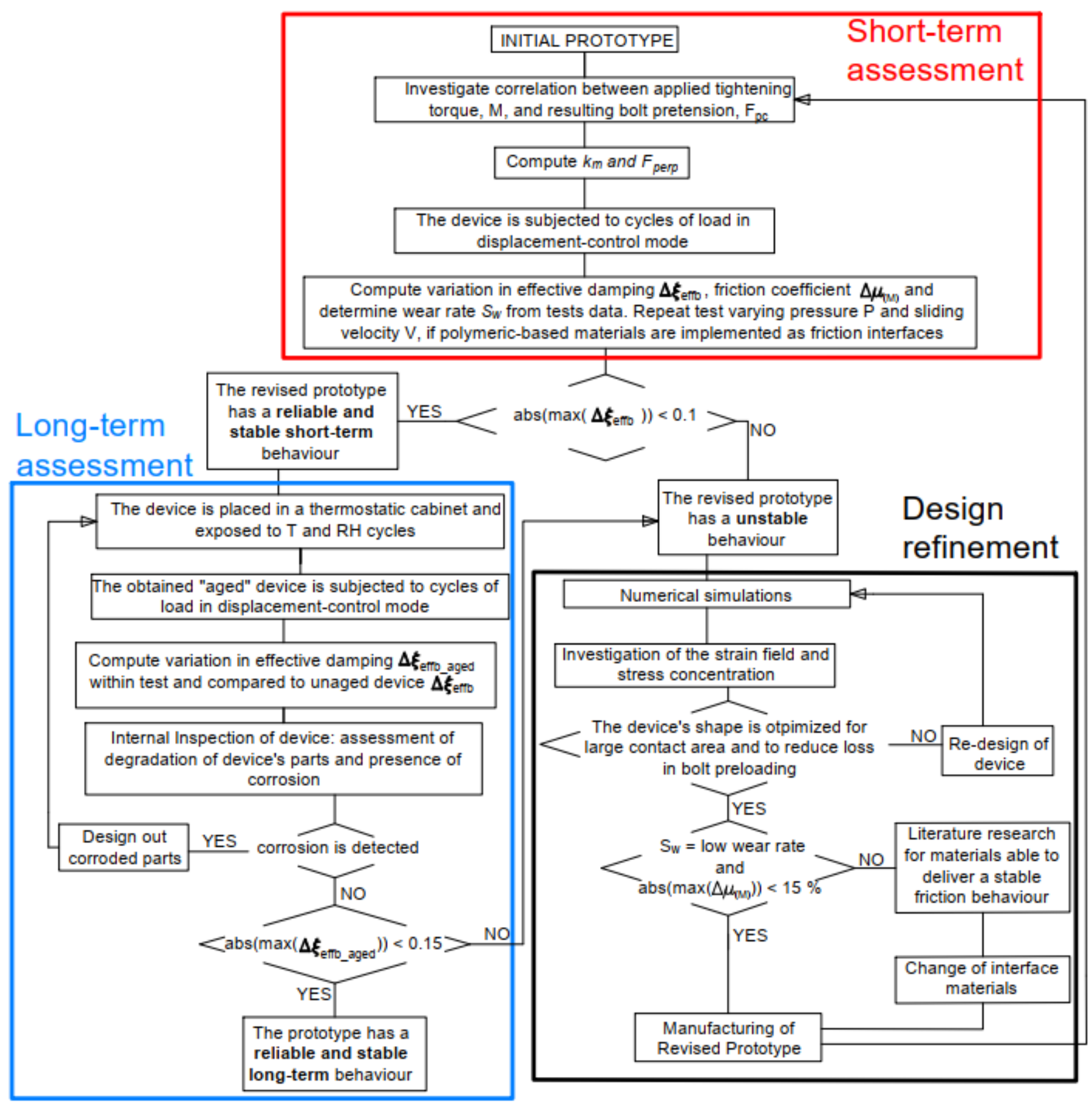

Fig. 3: Methodology for the assessment and refinement of friction-based dissipative devices.

\section{Short-term assessment - Characterisation of dissipative behaviour}

The short-term assessment aims to identify and quantify all the parameters that control the performance of the SteSq prototype. The reported results refer to the SteSq device tested in isolated configuration: the specimen is disconnected from the threaded bars that complete the dissipative system and $150 \mathrm{~mm}$-long threaded bars are screwed at both ends to allow the insertion of the device in the testing apparatus. The EN 15129 [13] and EN 1090-2 [43] are used for defining the testing protocols, as they provide guidance for performance acceptance of seismic dissipative systems and tightening operations of bolted unions.

\subsection{Quantification of $k_{m}$ for the specific device assembly}

The first task of the laboratory activity is to determine the clamping force $F_{p c}$ transferred to the fasteners during the tightening operation of the bolts. For the SteSq device, the torque method is applied by tightening the fasteners to a 
reference value of torque. The EN 1090-2 provides an empirical correlation between the reference tightening torque $M$ and the resulting nominal preload in the bolt $F_{p c(N)}$ :

$$
M=k_{m} d F_{p c(N)}
$$

where $d$ is the nominal diameter of the bolt and $K_{m}$ is assumed equal to 0.2 for non-lubricated bolt conditions. Eq.4 provides an approximation of the transferred preload as it is estimated that only about $10 \%$ of the tightening torque results in useful bolt tensioning. The remaining $90 \%$ is lost due to various forms of friction that occur during the tightening process [44]. In addition, the preload in the bolt can be inconsistent from fastener to fastener and depends on the type of nuts used. For these reasons, Eq 2 is not applicable to all situations and testing of actual fastener components is recommended to quantify $k_{m}$ for all critical-use applications [45].

Since the parameter $k_{m}$ includes all the factors affecting the relationship between applied bolt torque and resulting bolt tension, including number and layout of bolts and geometry of the objects to fasten, appropriate values were determined experimentally. The torque was applied in increasing steps of $5 \mathrm{Nm}$ to sets of four bolts by means of a dynamometric torque wrench. Each set is composed of four class 8.8 bolts with nominal diameter of $8 \mathrm{~mm}$, these being the fasteners used to apply the perpendicular load to the dissipative device.

The variation of the pretension transmitted throughout the test was recorded by two two donut load cells installed below the head of two bolts. In this way, the bolt pretension $F_{p c}$ and the tightening torque $M$ are unequivocally determined for each increment in torque. Two geometries were considered (see Fig. 4). Initially the pretension is recorded for bolts that go through a single plate (Fig. 4a) to determine the variation of transmitted bolt pretension when two different types of nut are considered, namely locking and normal nuts. Lock nuts are used to reduce the loss in bolt preloading that all bolted connection experience with time. A nylon ring is inserted inside the normal steel nut, imparting higher friction grip between the mating threads, thus increasing the friction force resisting the loosening effect [46].

In a second test the bolts are tightened to the dissipative device to determine the variation of bolt load due to the deformability of the plates forming the device during tightening (Fig. 4b). When the pre-tension is applied to deviating surfaces, a bending moment will be induced due to the inclination on one or both surfaces. As a result, the combined presence of axial force and bending moment may cause a higher stress levels under the bolt head [47]. Both locking and normal nuts are tested also for the second set of tests.

a)
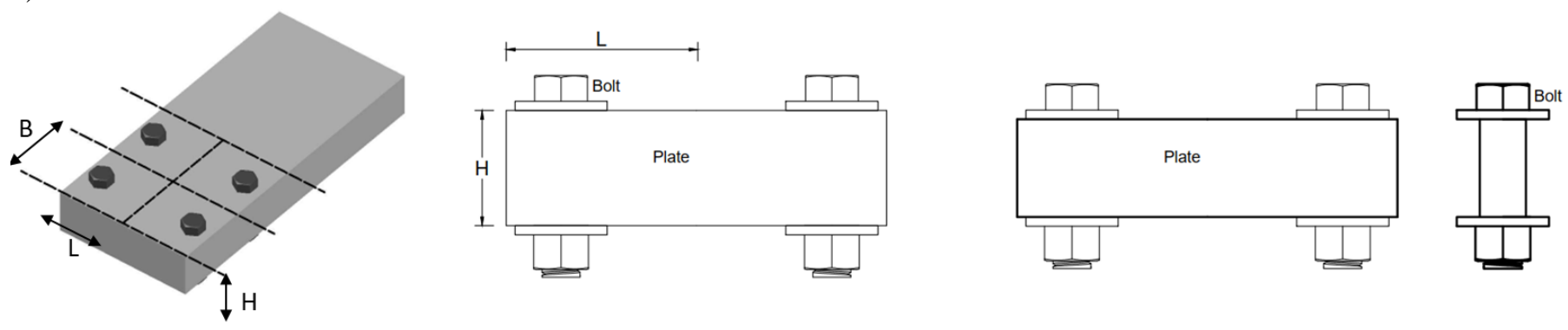

b)
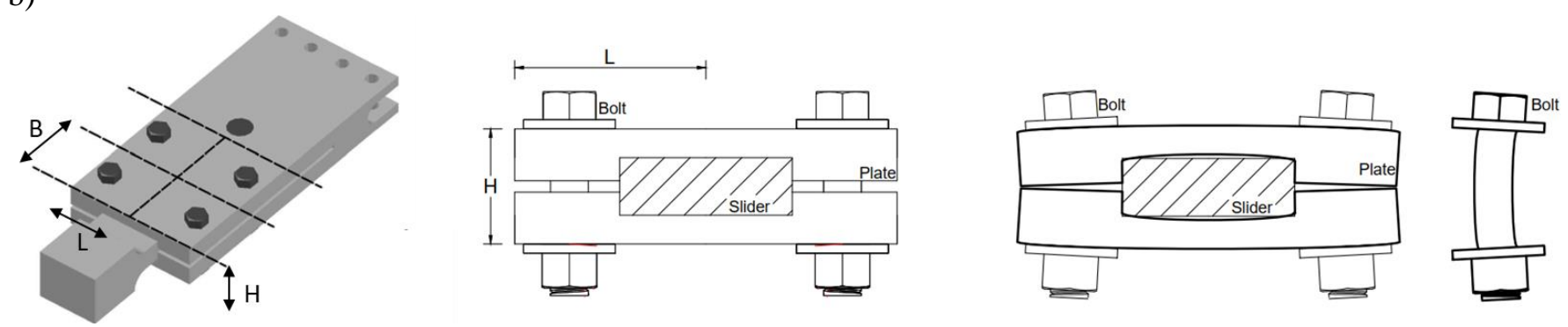

Fig. 4. Tested joint geometries: a) Single Plate (SP) geometry, b) SteSq Device (D) geometry. Undeformed and deformed shape of plates and bolts during tightening.

The predicted ultimate applicable tightening torque $M_{u}$ is computed from Eq. 5 as the torque required to achieve the proof load $F_{p l}$, equal to $70 \%$ of the nominal bolt strength, $f_{u b}$ : 


$$
M_{u}=k_{m} d F_{p l}=k_{m} d\left(0.7 f_{u b} A_{s}\right)=30 \mathrm{Nm}
$$

where $A_{s}$ is the nominal stress area of the bolt and $K_{m}$ is equal to 0.2. Fig. 5 shows the values of the bolt load recorded for each geometry and type of nut, where the points represent the mean value of the set of bolts.

It was found that the mean values deviate from the nominal values obtained by applying Eq. 5, which are also shown in Fig. 5 as reference. For the single plate (SP) configuration, a linear correlation between the applied tightening torque and the recorded bolt preload is obtained, as shown in Fig. 5a. Moreover, both Fig. 5a and Fig. 5b show that the use of lock nuts causes a reduction of bolt load in the range of $10-15 \%$ with respect to normal nuts. This is because during the tightening process the lock nuts exhibit higher friction with the threads so that a smaller portion of the torque is transmitted to the bolt's stem as pretension. Regarding the tests carried out on the device configuration, Fig. 5b highlights that the geometry determines a loss in the bolt preload for increasing values of torque.

a)

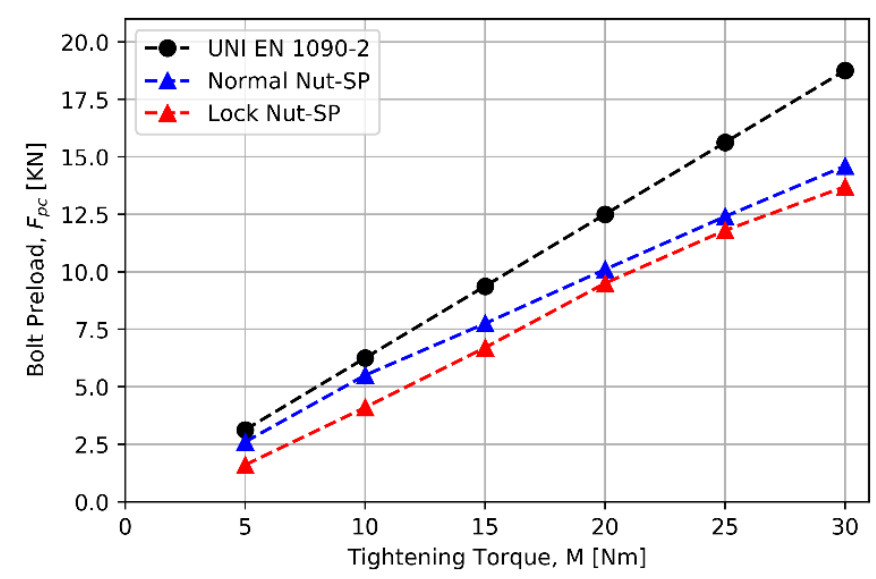

b)

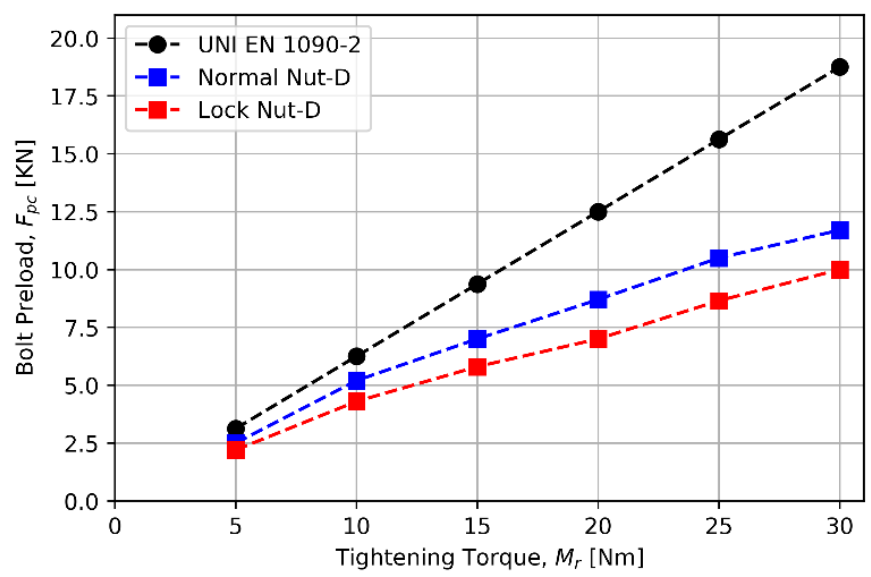

Fig. 5. Bolt pretension when normal nuts and lock nuts are employed on a) single plate geometry (SP) and b) device geometry (D).

A simplified model can be implemented to consider the behaviour of the device and compute the loss of bolt preload. With reference to Fig. 6a, a beam-model of the upper plate is created, following the middle-surface of the plate. The element $\mathrm{ABCD}$ represents half of the upper plate, the vertical spring at point A represents the response of the bolt along its longitudinal axis, while the horizontal spring at $\mathrm{A}$ and the spring at point $\mathrm{C}$ represent the axial stiffness of the slider in the horizonal and vertical direction, respectively.

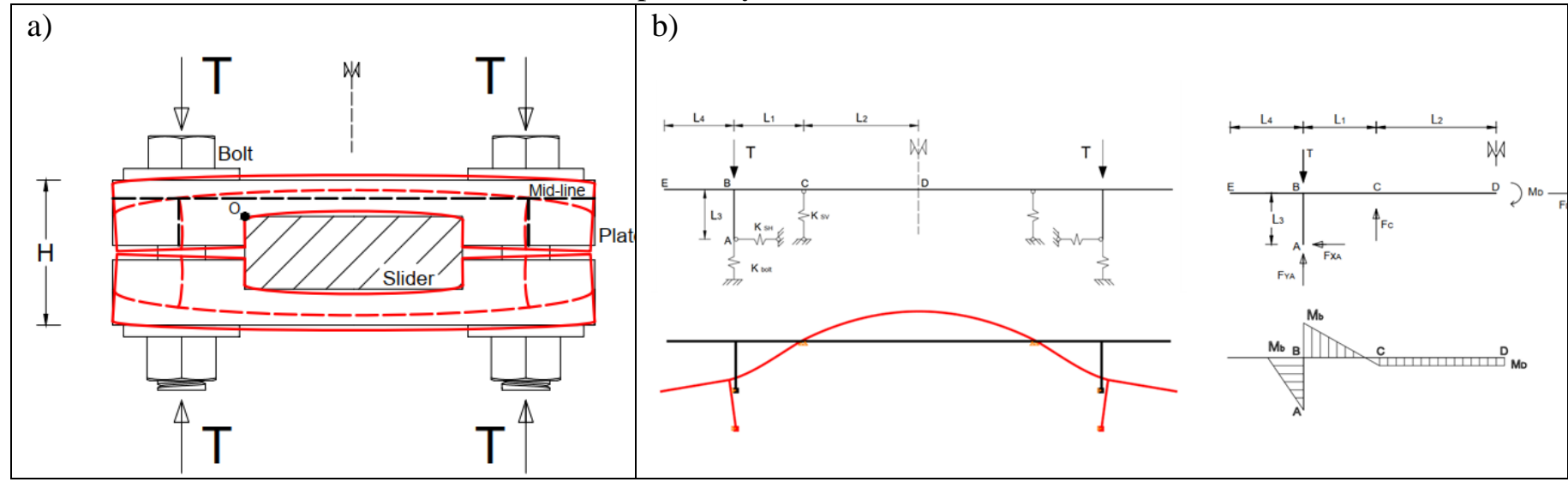

Fig. 6. a) Cross section of SteSq device, mid-line of clamping plate (dotted line) b) Numerical model of the clamping plate, deformed shape, free body diagram and diagram of bending moment

An increasing vertical force is applied at point B to study the deformation of the model during the tightening. Fig. $6 \mathrm{~b}$ illustrates the obtained free body diagram and the diagrams of bending moment which increases linearly along element $\mathrm{AB}$ and it is maximum at point $\mathrm{B}$.

The forces acting on the bolt can be derived from element $\mathrm{AB}$ and are reported in Fig. 7a. With reference to Fig. 7 the resulting tensile stress is the combination of the axial load and the bending moment and it is maximum under the head of the bolt (Section A-A) where the contribution of the bending moment is maximum. The shear stresses are negligible compared to the tensile stress generated by the combined presence of axial force and bending moment under the bolt 
head. For values of bolt preload greater than $7.5 \mathrm{KN}$, the bending moment causes the tensile stress to exceed the yielding limit, causing localized plasticity. Assuming a perfectly plastic behaviour of the bolt's material beyond the yielding point, a portion of traction force is lost with respect to an elastic model.

a)

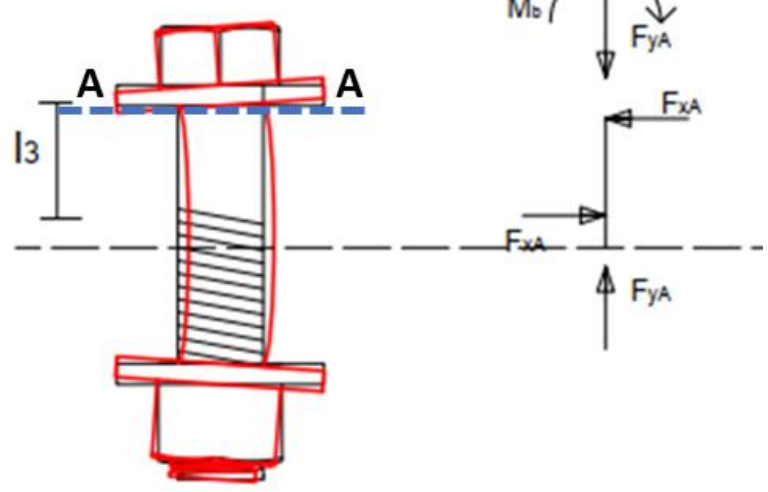

b)

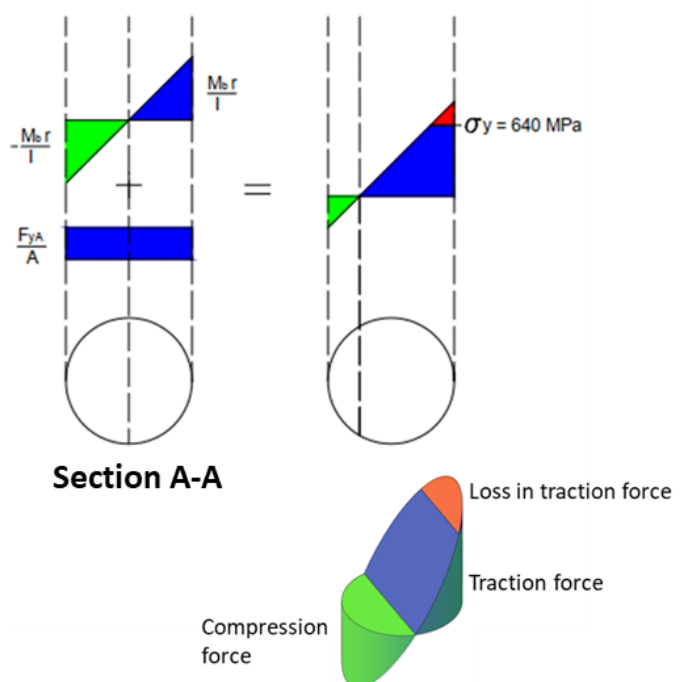

Fig. 7. a) Forces acting on the bolt's shank, b) Tensile stress under the bolt's head

The non-linear evolution of the analytical model is compared with the experimental values recorded for the device configuration in Fig. 8a. In general, the correspondence between the numerical model and the experimental trend is well captured. Initially, the numerical model displays a liner trend, confirming that the bolts remain in their elastic phase for torque reference values up to $15 \mathrm{Nm}$. According to BS EN 1090-2, this value can be considered as maximum permissible value of torque for the device, as it induces a tensile stress equal to $90 \%$ of the yielding limit [48]. For larger values of torque, the model displays a non-linear behaviour reflecting the loss in bolt preload due to the local yielding at the bolt's head, The loss is well captured by the numerical model which assumes a bilinear constitutive law, shown in Fig. 8b, obtained from the values of nominal ultimate stress and deformation for bolt class 8.8 [49].
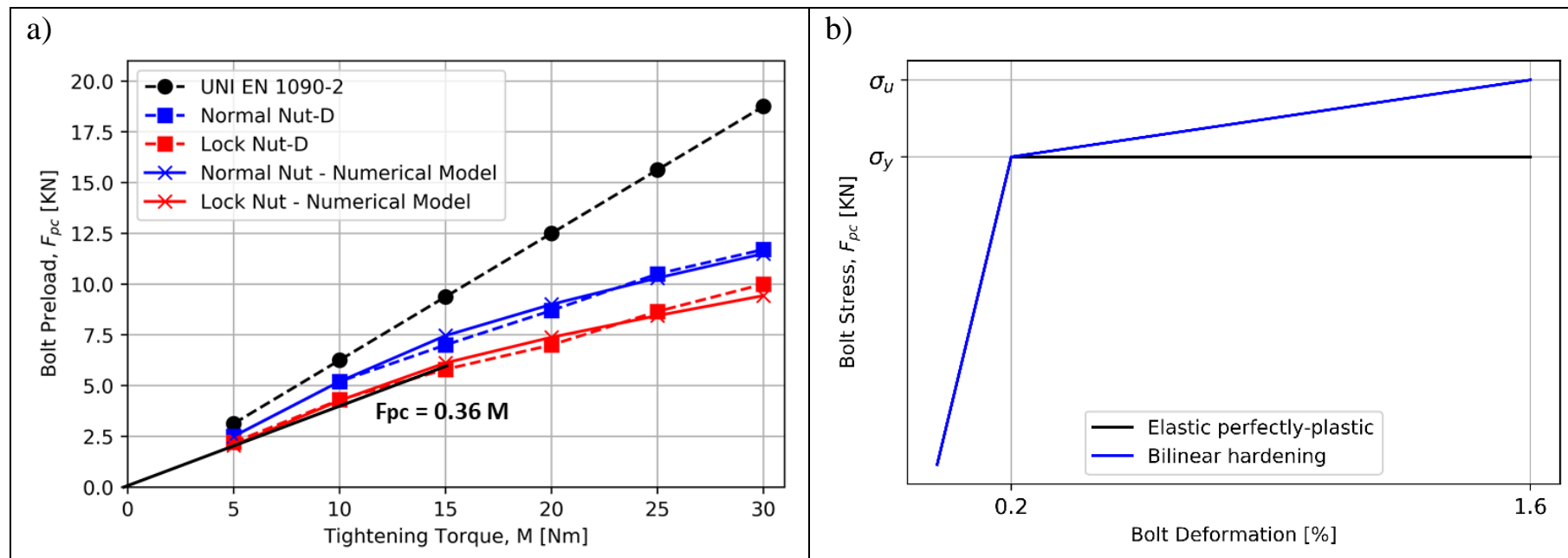

Fig. 8. Correspondence between numerical model and experimental evidence: a) perfectly plastic behaviour of steel, b) bilinear behaviour of steel

To keep consistency with the linear formulation of Eq. 5, the linear regression over the measurement on lock nuts, shown in Fig. 8a, is used to obtain an empirical equation relating the applied torque - up to the maximum permissible value of torque $(15 \mathrm{Nm})$ - to the nominal bolt pretension:

$$
F_{p c(N)}=0.36 M
$$

Comparing Eq. 6 to Eq. 4 and considering that the nominal diameter of the bolts is $8 \mathrm{~mm}$ :

$$
F_{p c(N)}=\frac{M}{k_{m} d}=\frac{M}{k_{m} 8}=0.36 M
$$


Which allows to compute the specific value of $\mathrm{k}_{\mathrm{m}}$ applicable to the device as:

$$
k_{m}=0.35
$$

Once the bolt preloading $\mathrm{F}_{\mathrm{pc}}$ is determined for each value of tightening torque, the nominal value of perpendicular force $F_{\text {perp (N) }}$ applied to the sliding surfaces is determined as:

$$
F_{\text {perp }(\mathrm{N})}=m F_{p c(N)}
$$

where $m$ is the number of tightened bolts.

\subsection{Assessing the stability of the effective viscous damping $\xi_{\text {eff }}$}

The objective of the second testing activity is to evaluate the performance of the device under cyclic loading. The test layout consists of one full-scale SteSq prototype connected to a universal testing machine, which imposes a relative motion to the slider according to a sinusoidal displacement history. Before the motion is initiated, the bolts gripping the assembly are tightened to a reference value of torque, which determines a nominal value of perpendicular force $F_{\text {perp }(\mathrm{N})}$ according to Eq. 6 and Eq. 9. Several tests are performed on the same device, in sequence, for values of tightening torque increasing by $5 \mathrm{Nm}$ up to the value of maximum allowable torque as determined in the previous section. Each test consists of 20 cycles of sinusoidal displacement to a maximum amplitude of $+-5 \mathrm{~mm}$. The pretension of two bolts, $\mathrm{F}_{\mathrm{pc} 1}$ and $\mathrm{F}_{\mathrm{pc} 2}$, is monitored throughout the duration of the test. The measured variation of applied load $F_{\text {perp }(\mathrm{M})}$ is computed as:

$$
F_{\text {perp }(\mathrm{M})}=m F_{p c(M)}=m \frac{F_{p c 1}+F_{p c 2}}{n_{l c}}
$$

where $m$ is the number of tightened bolts and $n_{l c}$ is the number of load cells. During the tightening operations the accuracy $\beta$ of the measured value of perpendicular force, $F_{\text {perp (M) }}$ is computed as:

$$
\beta=F_{\text {perp }(M)} / F_{\text {perp }(N)}
$$

Among the tightening methods proposed by EN1090-2 (combined, torque, tension control), the torque method shall be capable of achieving $\pm 95 \%$ reliability [50], thus a tolerance of $5 \%$ between nominal and measured force is allowed (see Table 1).

Besides the pressure cells, the testing apparatus also comprises two LVDTs to measure the displacement $d_{r}$ of the slider relative to the fixed sleeve of the device and two strain gauges to measure local strains where the greater deformations are expected, namely near the bolts and the locking end, as shown in Fig. 9.
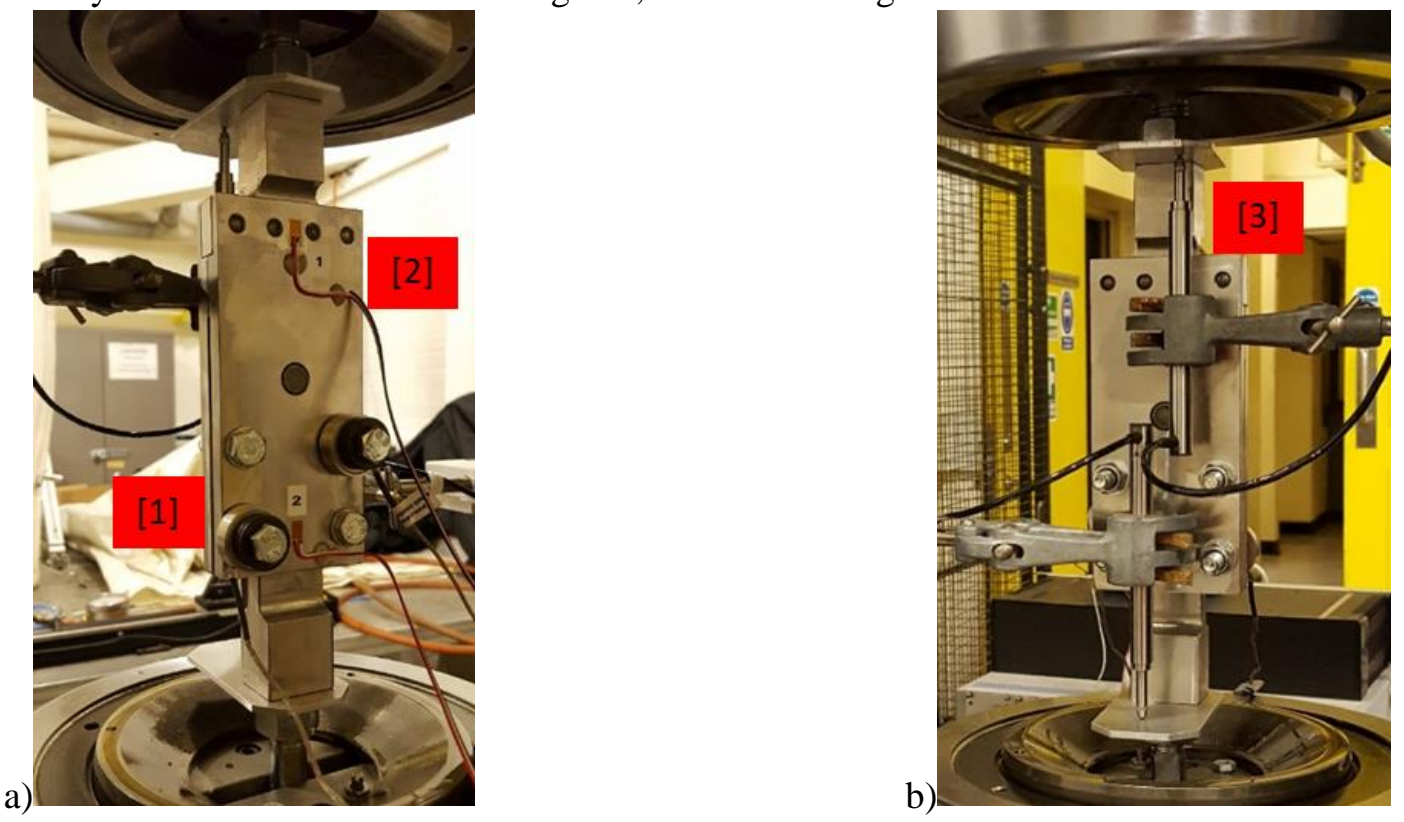

Fig. 9. Testing apparatus: [1] load cells, [2] strain gauges, [3] LVDTs 
EN 15129 [13] is taken as reference for this testing activity, as it provides the testing requirements for the verification of consistency of seismic devices' performance. According to the guidelines, the sinusoidal displacement history is defined as follows:

$$
d_{r}(\mathrm{t})=d_{0} \sin \left(2 \pi f_{0} t\right)
$$

where $d_{0}$ is the peak stroke (set at $\pm 5 \mathrm{~mm}$ ), $f_{0}$ is the loading frequency (set at $0.1 \mathrm{~Hz}$ ) and $t$ is the time in seconds.

The slippage force $F_{\text {slip (M) }}$ that determines the relative motion of the slider is measured by a load cell in series to the device and the values of friction coefficient are obtained as:

$$
\mu_{M}=\frac{F_{\text {slip }(\mathrm{M})}}{n F_{\text {perp }(M)}}
$$

The design value of friction coefficient $\mu_{\mathrm{D}}$ is obtained as the mean value of $\mu_{\mathrm{M}}$ recorded during over 20 cycles of load and the design force of the device is determined as follows:

$$
V_{D}=\mu_{\mathrm{D}} n F_{\text {perp }(\mathrm{N})}
$$

In Eq. 13 the number of friction surfaces $n$ is one of the three parameters that determines the value of friction coefficient. In their simplest design, bolted-slotted devices present two plates clamped on friction pads by pre-stressed bolts. Given their geometry only the upper and lower surfaces of the friction pad are pre-stressed and involved in the sliding motion. Therefore, the number of friction surfaces is equal to $2(n=2)$, as reported by several authors $[25,26,40]$.

On the other hand, the geometry of the SteSq device makes the external plates bend around the slider element when the bolts are tightened. Thus, the lateral surfaces are acted upon by a positive pressure and contribute to the resistance to sliding exerted through friction, together with the upper and lower surfaces (see Fig. 14 for a representation of the lateral contact pressure on the slider). Therefore, to compute the value of friction coefficient four contact surfaces are considered in Eq. $13(\mathrm{n}=4)$.

According to EN 15129 the hysteretic capacity of the device can be computed considering the effective viscous damping for each cycle:

$$
\xi_{\text {effb }}=\frac{W\left(d_{b}\right)}{2 \pi V_{D} d_{b}}=\frac{4 F_{\text {slip }(\mathrm{M})} d_{b}}{2 \pi V_{D} d_{b}}=\frac{4 F_{\text {slip }(\mathrm{M})}}{2 \pi V_{D}}
$$

Where $W\left(d_{b}\right)$ is the energy dissipated by the device during each cycle, with maximum displacement equal to $d_{b}$, and $V_{D}$ is the design force of the device. To assure a stable behaviour under cyclic loading, EN 15129 limits the effective damping variation in a series of load cycles relevant to the same displacement as follows:

$$
\Delta \xi_{e f f b}=\frac{\left|\xi_{e f f b, i}-\xi_{e f f b, 3}\right|}{\xi_{e f f b, 3}}<0.1
$$

where subscripts 3 and $i$ relate to quantities at the $3^{\text {rd }}$ and $i^{\text {th }}$ load cycle of an experimental test, with $\mathrm{i} \geq 3$.

Results are presented in terms of load displacement history for each increment of torque applied to the bolt. The loaddisplacement loops shown in Fig. 10a have a regular rectangular shape as expected for a friction-based sliding device, but a change in slippage load is noticeable between successive cycles due to progressive wear of surfaces in contact. The presence of debris particles lost by the worn-out surfaces in contact remains within the assembly, thus creating additional resistance to slide. As a result, $F_{\text {perp }(M)}$ increases from the initial imposed value. For the case of $15 \mathrm{Nm}$, the measured perpendicular force displays the larger variation, initially increasing by $6 \%$ in 11 cycles and then decreasing by $10 \%$, due to loss in bolt preloading, as shown in Fig. 10b, where $F_{\text {perp }(M)}$ is normalized with respect to the nominal value $F_{\text {perp }(N)}$. The value of $\beta$ for the first cycle, as computed from Eq. 11, is above the target of $95 \%$ for 5 and $10 \mathrm{Nm}$ and just below the threshold $(\beta=93.3 \%$ ) for $15 \mathrm{Nm}$, possibly due to the instantaneous loss of pre-loading between the tightening phase and the application of the cyclic loading protocol. 
a)

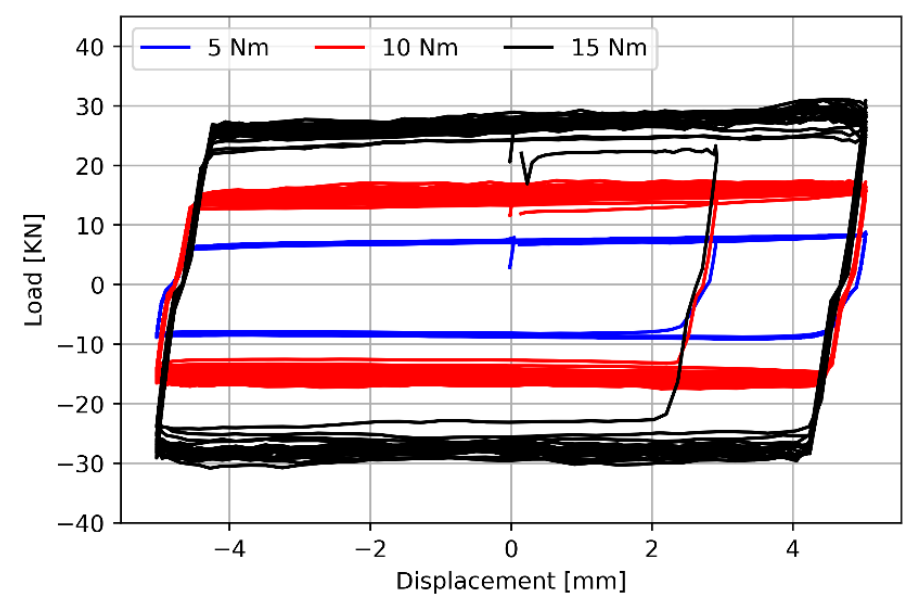

b)

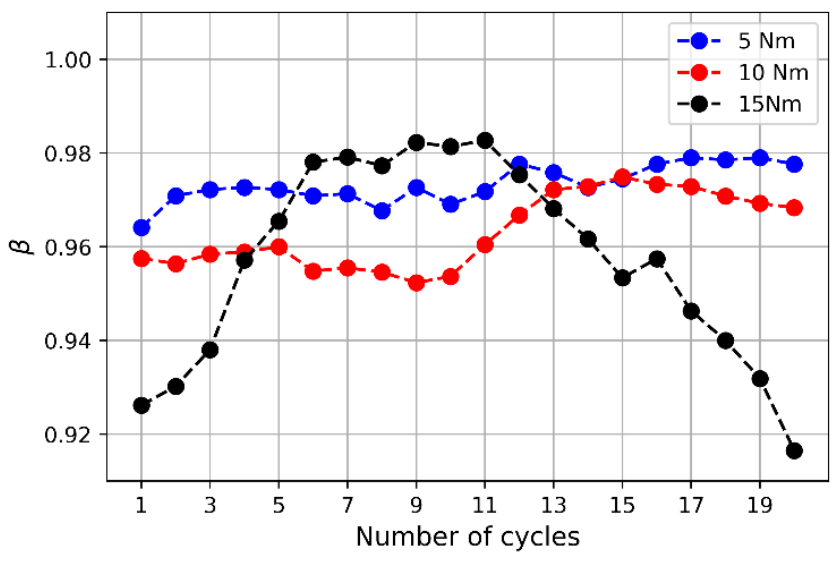

Fig. 10. Test results for nominal bolt torque of 5,10 and $15 \mathrm{Nm}$ over 20 cycles of load: a) load displacement loops, b) maximum values of $F_{\text {perp (M) }}$ in each cycle, normalized to the nominal value $F_{\text {perp (N) }}$.

The repeated rubbing of the plates also produces surface roughness, thus increasing friction. The initial value of friction $\mu_{0}(\mathrm{M})$ is computed with respect to the first cycle of load, for each value of torque imposed. The increase in $\mu_{(\mathrm{M})}$ with respect to the initial value is shown in Fig. 11a, for each value of torque imposed, while the $\mu_{\mathrm{D}}$ value and $\max \left(\mu_{(\mathrm{M})}\right)$ values are reported in Table 1. The maximum variation of $\mu_{(\mathrm{M})}$ from $\mu_{\mathrm{D}}$ over 20 cycles is obtained for $\mathrm{M}=10 \mathrm{Nm}$ $\left(\max \left(\Delta \mu_{(\mathrm{M})}\right)=15 \%\right)$, while the absolute maximum value of friction coefficient, $\mu_{\max }=0.39$, is obtained for a nominal torque of $15 \mathrm{Nm}$ after 57 cycles of load.

a)

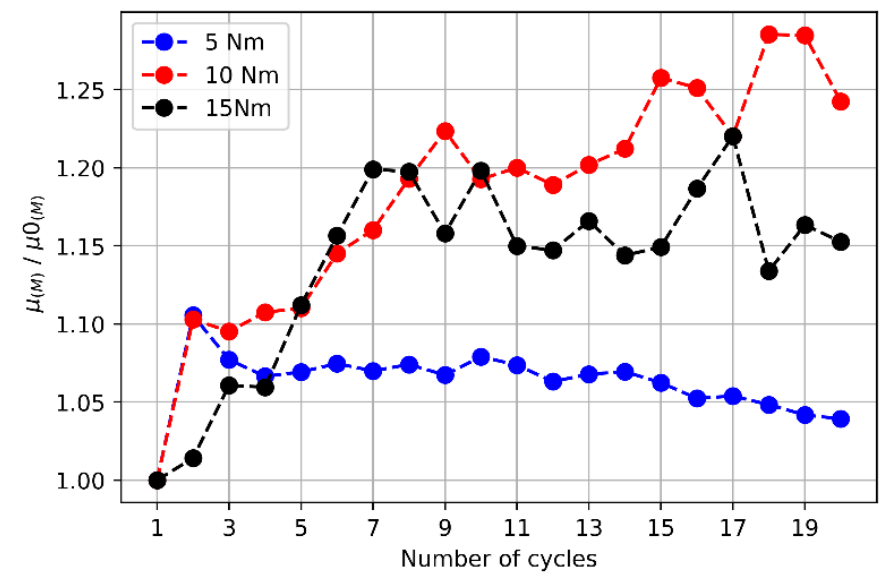

b)

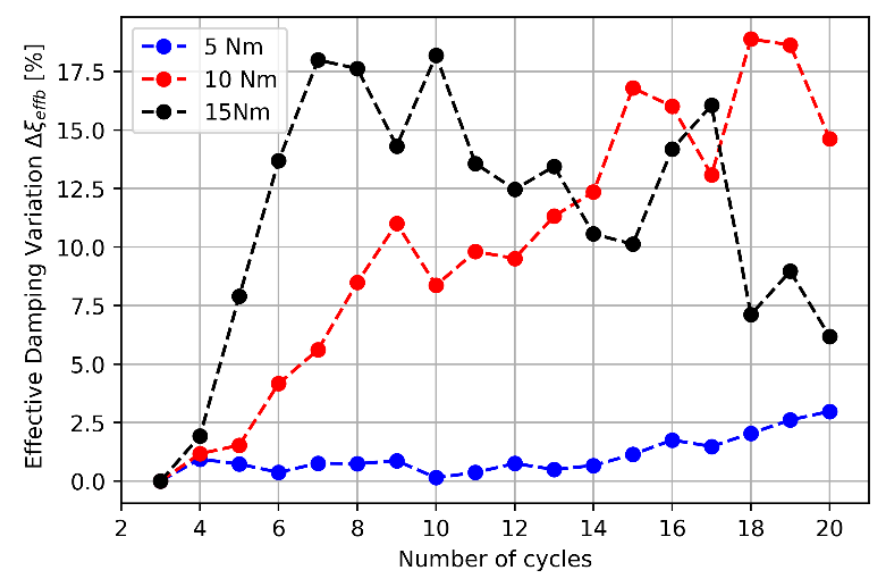

Fig. 11. a) Variation of friction coefficient $\mu_{(\mathrm{M})}$ with number of cycles, and $b$ ) of effective damping.

The variation of effective damping (see Fig. 11b) is calculated according to Eq. 15. The combined effect of additional pressure and interlocking phenomenon due to surface roughness determines that subsequent tests require a greater slippage force to overcome the additional mechanical resistance. Moreover, the range of values of sliding force widens with increasing values of initial tightening torque. According to EN 15129, the absolute variation of effective damping over the series of cyclic testing, should not differ by more than $10 \%$ from the value of effective damping recorded at the $3^{\text {rd }}$ cycle. As showed in Fig. 11b, for M equal to 10 and $15 \mathrm{Nm}$,

$$
\operatorname{abs}\left(\max \left(\Delta \xi_{\text {effb }}\right)>0.18\right.
$$

while for $\mathrm{M}=5 \mathrm{Nm}$ it is below the threshold value. Nonetheless, the slippage force recorded at $5 \mathrm{Nm}$ would determine a small contribution in terms of dissipated energy, making the device unfit for a seismic upgrade.

Table 1. Results of test activity on SteSq prototype

\begin{tabular}{|l|l|l|l|r|}
\hline \multirow{2}{*}{ Variable } & \multirow{2}{*}{ Symbol } & \multicolumn{3}{|c|}{ Reference torque values } \\
\cline { 4 - 5 } & & 5 & 10 & 15 \\
\hline
\end{tabular}




\begin{tabular}{|c|c|c|c|c|}
\hline & & {$[\mathrm{Nm}]$} & {$[\mathrm{Nm}]$} & {$[\mathrm{Nm}]$} \\
\hline Nominal bolt preload & $\mathrm{F} \mathrm{pc}(\mathrm{N})$ & 1.79 & 3.57 & 5.36 \\
\hline Measured bolt preload & $\mathrm{F} \mathrm{pc}(\mathrm{M})$ & 1.75 & 3.45 & 5 \\
\hline Nominal perpendicular force & $\mathrm{F}$ perp $(\mathrm{N})$ & 7.14 & 14.3 & 21.4 \\
\hline Measured perpendicular force & $\mathrm{F} \mathrm{perp} \mathrm{(M)}$ & 7 & 13.8 & 20 \\
\hline Accuracy of initial F perp & $\beta$ & $98 \%$ & $96.60 \%$ & $93.33 \%$ \\
\hline Initial friction coefficient & $\mu \mathrm{N}(\mathrm{M})$ & 0.26 & 0.26 & 0.32 \\
\hline Max friction coefficient & $\mu \max (\mathrm{M})$ & 0.288 & 0.34 & 0.392 \\
\hline Design friction coefficient & $\mu_{\mathrm{D}}$ & 0.28 & 0.31 & 0.36 \\
\hline Max variability of $\mu$ (M) & $\max (\Delta \mu(\mathrm{M}))$ & $7 \%$ & $15 \%$ & $11 \%$ \\
\hline Design force & $\mathrm{V}_{\mathrm{D}}$ & 8 & 17.7 & 30.9 \\
\hline Slippage Force at $3^{\text {rd }} \mathrm{cycle}$ & $\mathrm{F}_{\mathrm{sl}, 3}$ & 7.87 & 16 & 26.3 \\
\hline Max Slippage Force & $\mathrm{F} \mathrm{sl}(\mathrm{max})$ & 8.00 & 19.00 & 31.10 \\
\hline Min Slippage Force & $\mathrm{F} \mathrm{sl}(\min )$ & 7.24 & 14.5 & 24.4 \\
\hline Effective Damping at $3^{\text {rd }} \mathrm{cycle}$ & $\xi_{\text {effb, }}$ & 0.624 & 0.56 & 0.52 \\
\hline Max variability of Eff. Damping & $\max \left(\Delta \xi_{\text {effb }}\right)$ & $2.97 \%$ & $18.80 \%$ & $18.18 \%$ \\
\hline
\end{tabular}

The visual inspections conducted on the dissipative devices after 60 cycles confirms that both the horizontal and vertical faces of the slider exhibited evidence of resistance through friction, as shown by the severe signs of wear (see Fig. 12), also confirming that the number of surfaces involved in the action is 4 . Nonetheless, the abrasion is visible in proximity of the edges of the frictional plates while no signs of wear are present in their central portion. This means that the geometry of the sample produces a non-homogenous distribution of pressure over the frictional plates, which appear to be only partially in contact as already highlighted in section 4.1. The reduced contact area leads to high-stress concentration, which explains the excessive wear and tear, the resulting increase in apparent friction coefficient $\mu_{\mathrm{M}}$ and the variations of the hysteretic loops among loading cycles.

The depth of the degradation signs was measured to a maximum value of $1.5 \mathrm{~mm}$. Given the abrasion depth and the computed maximum variation of $\mu_{(\mathrm{M})}$, the wear rate $S_{\mathrm{w}}$ is deemed "severe with high wear rate".

a)

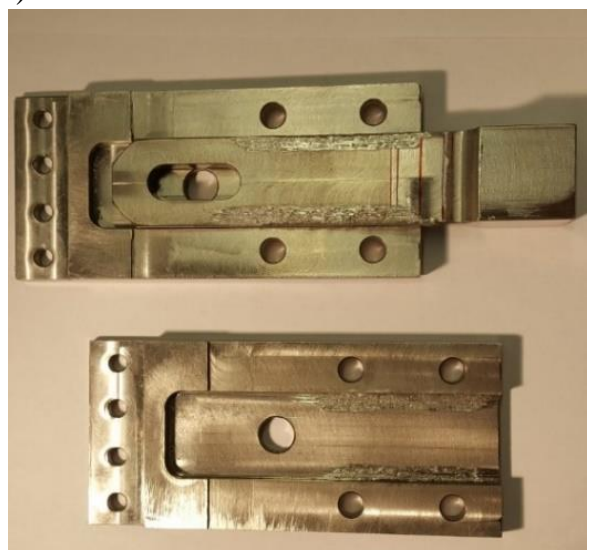

b)

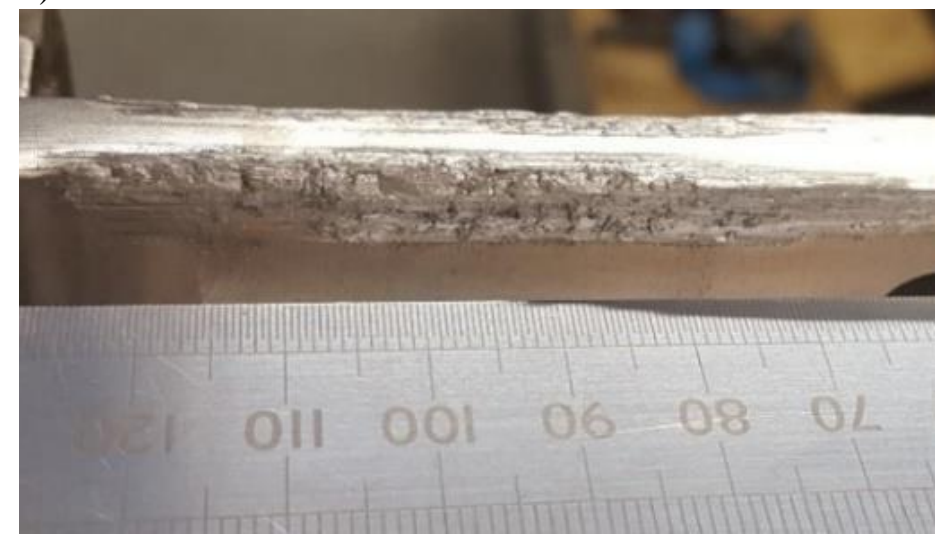

Fig. 12: Superficial wearing of the frictional device: a) the assembly; b) particular of the slider

\section{Design refinement}

The testing activities highlights several shortfalls for prototype SteSq. During the tightening operations, its geometry induces high tensile stress under the bolts' head, leading to localized plasticity for reference values of torque greater than $15 \mathrm{Nm}$. This leads to preload-loss directly after tightening and to a non-linear correlation between applied torque and effective bolt preload. To avoid this, the maximum allowable torque is halved compared to a configuration where the plates remain flat during tightening, thus drastically reducing the maximum friction resistance the device can produce.

The vibrations induced during cyclic loading did not cause significant bolt loosening, as the bolt preload variation is within a $\pm 10 \%$ threshold. Conversely, the friction coefficient displayed a hardening behaviour, significantly increasing from the recorded initial value. As expected in the case of steel-on-steel friction [35], severe wear with high wear rate 
is registered, and the removal of slivers from both surfaces determines surface roughness. This effect is worsened by larger pressure acting on the plates and three design values of friction coefficients $\mu_{D}$ are obtained, namely $0.28,0.31$ and 0.37 for 5,10 and $15 \mathrm{Nm}$ of reference torque respectively. Consequently, the slippage load shows an increasing trend over 20 cycles of load, and the variability of effective damping is greater than the recommended value in EN 15129.

According to the methodology proposed in Fig. 3, the SteSq prototype failed the reliability condition for the short-term assessment, therefore different design solutions are analysed to improve its behaviour according to the tests' results.

\subsection{Numerical model of SteSq}

A 3D numerical model of the SteSq prototype is developed using ABAQUS software [51] to allow for a detailed representation of all prototype's parts to investigate the strain field produced during testing.

The numerical model comprises three parts, representing the slider, the clamping plates and the tightening bolts (Fig. 13). Three-dimensional volume elements (C3D8R) are used for the mesh since a complete and accurate threedimensional stress/strain distribution is critical to interpret the phenomena observed during the tests. Boundary conditions are imposed to reproduce the experimental testing conditions: the two external plates are fully restrained, and the slider is allowed to move only in the direction of its longitudinal axis (x-axis), even though it is acknowledged that the tolerances present in the physical specimens would allow small relative motion of the parts in the two transversal direction.
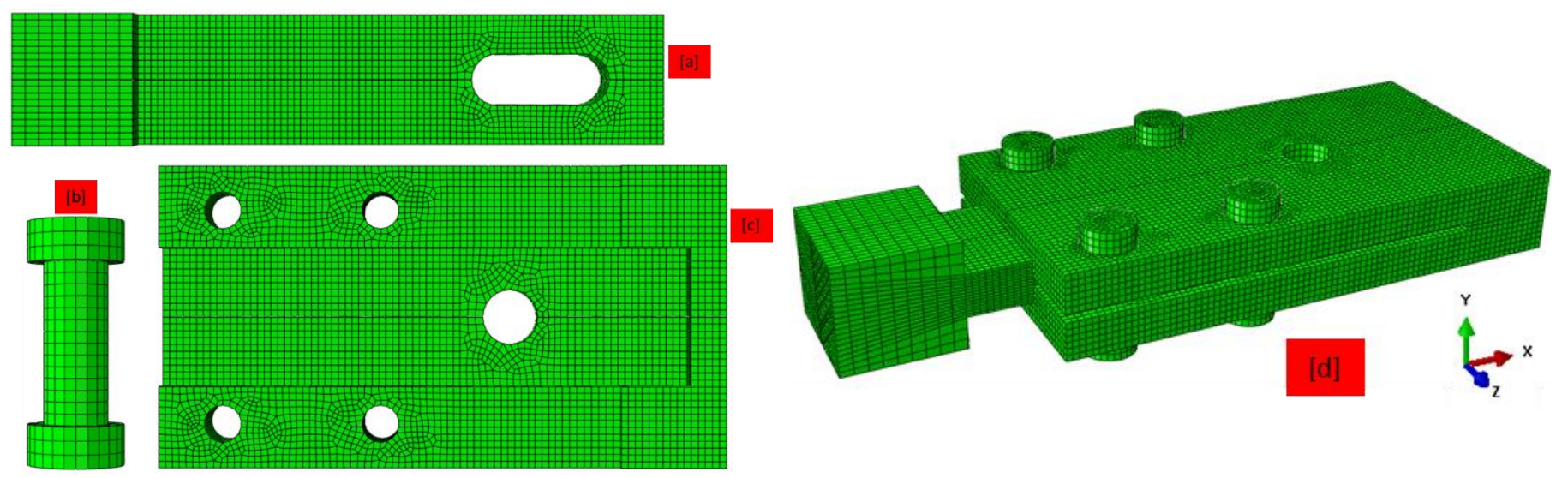

Fig. 13. Parts of the numerical model, [a] slider, [b] bolt, [c] frictional/clamping plate, and [d] assembly.

The interaction properties between the surfaces in contact are defined by assigning a friction coefficient to all parts in contact. The same displacement time-histories defined during the testing campaigns 2 are used as input for the FE models, thus ensuring correspondence between experimental and computational tests.

To reflect the physical specimen's conditions, the $F_{\text {perp }(M)}$ is applied by modelling four bolts and applying a concentrated force to each bolt head, as per ABAQUS specific routine. The $F_{p c}$ assigned to each bolt-like part is $5 \mathrm{KN}$, thus simulating the $F_{\text {perp }}(M)=20 \mathrm{KN}$, measured for the test with $15 \mathrm{Nm}$ of nominal bolt torque. This test was selected for the simulation because the device displayed less variability compared to the $10 \mathrm{Nm}$ case, while providing almost 3 times the energy dissipation capacity obtained by the test with $5 \mathrm{Nm}$ torque. Moreover, for the $15 \mathrm{Nm}$ test, $\mu$ rapidly increases during the first 5 cycles, (from 0.32 to 0.36 ) and then remains quite stable throughout the rest of the test. Therefore, the value of friction coefficient is set to 0.36 to capture the "stable" phase and to 0.32 to reproduce the initial state of the device. These values are reported in Table 1 as $\mu_{D}$ and $\mu_{0}$ (M) for $M=15 \mathrm{Nm}$.

The analysis involves two steps. First, a static analysis is performed to apply the bolt loads within the first time-step. In the second step, a sinusoidal displacement is imposed to the sliding part to reproduce the cyclic motion of the experimental activity (step two). In Fig. 14a the device is presented as cut along section A-A and the slider is hidden to ease the visualization of the deformed shape of the sleeve plates at the end of Step 1: it is evident that the applied bolt's pressure produces a bending of the two external plates around the internal sliding element, confirming the output of the simplified model proposed in section 4.1. and Fig. 6. As shown in Fig. 14the bending of the plates concentrates the contact area around the edges of the slider, producing a distribution of longitudinal shear stresses, computed in the second step, corresponding to the worn surfaces of the test shown in Fig. 12. 
a) Step 1

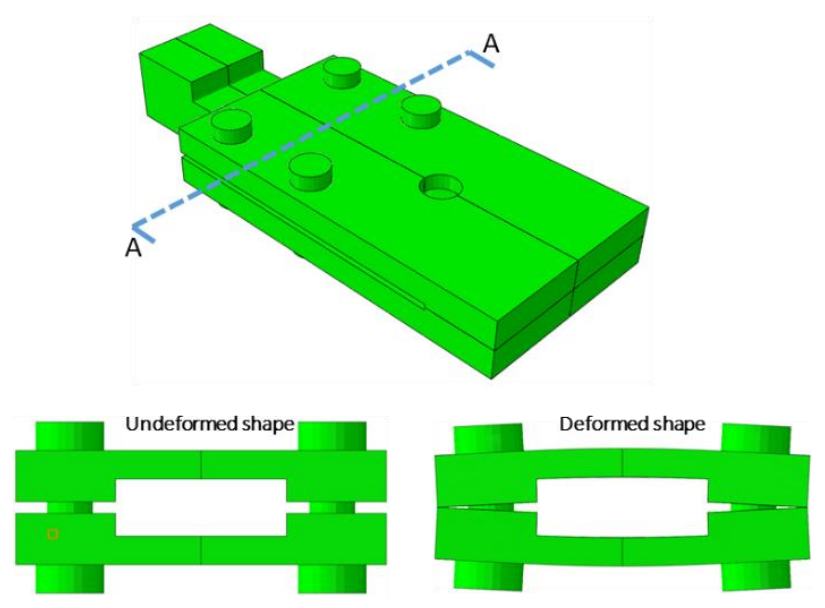

b) Step 2

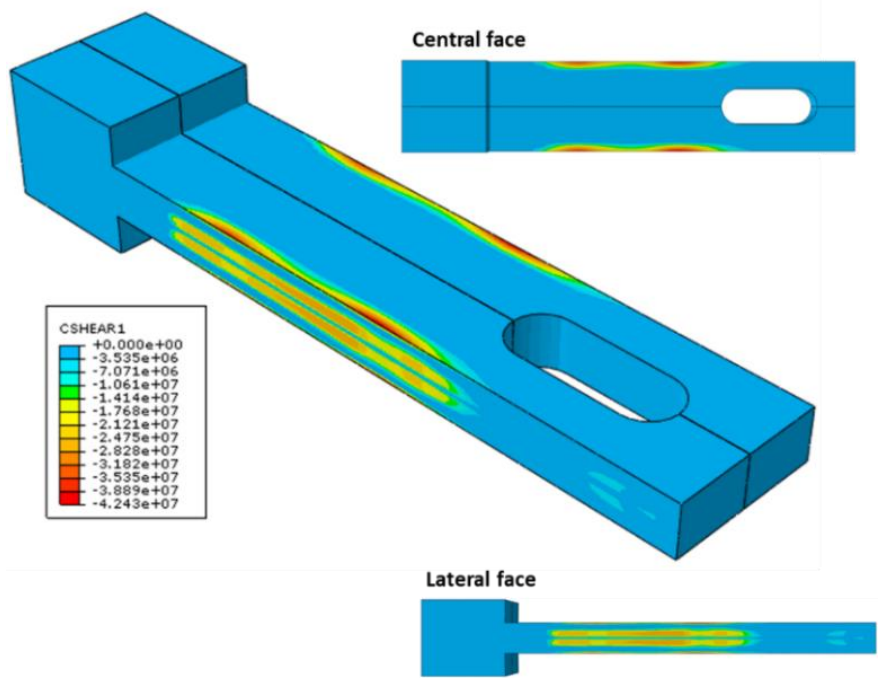

Fig. 14. Results of numerical analysis: a) deformed shape following the tightening of the bolt for a value of $4.7 \mathrm{KN}$, b) distribution of shear stresses over the sliding element.

The sliding force required to exceed the friction resistance can be computed integrating the shear stress over the external surface of the slider. It was found that the friction force developed over the lateral faces $(19.4 \mathrm{KN})$ is almost twice the one on the horizontal surfaces $(10.1 \mathrm{KN})$. The large contribution of the lateral faces to $F_{s l ~ n u m}=29.8 \mathrm{KN}$, confirms that four pair of contact surfaces should be considered for the prototype ( $n=4$ in Eq. 2 and 13). The value of $F_{s l}$ is in good agreement with the maximum value of slippage force $\mathrm{F}_{\mathrm{sl}(\max )}=31.1 \mathrm{kN}$ measured experimentally for $\mathrm{M}=15 \mathrm{Nm}$.

Fig. 15a shows the numerical simulation's load-displacement loops for 20 cycles of imposed periodic displacement for $\mu=0.36$. While the hardening behaviour and the slack at the inversion of load, are not reproduced, the values of slippage force for 0.36 is within $6 \%$ of the experimental maximum value. Therefore, this limit values of $\mu=0.36$ can be considered as the upper bound that identifies the device's operability at a fixed perpendicular pressure.

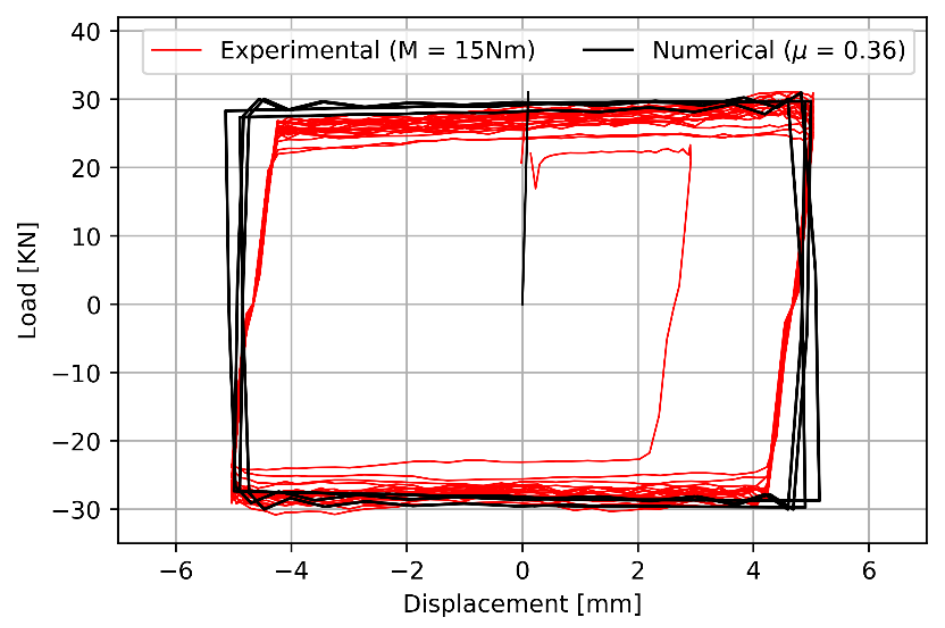

Fig. 15. Comparison between computational a) and experimental results b)

\subsection{Second generation prototypes}

The results of the numerical analysis confirm the validity of the conceptual design of the device, but also the need for substantial refinements, to reduce wearing off of the surface and improve stability of the effective damping. Such observations have informed the design of a second prototype, whereby the basic concept is maintained, however the shape is altered to replace the internal flat slider and the sharp edges of the sleeves which caused the concentration of stresses.

The result of the new design is a cylindrical shape for the slider and its housing within the sleeve, as shown in cross section in Fig. 16a. The shape is optimized reducing the distance, $e$, between the bolt's axial line and the extreme point 
of contact, namely point $O$ in Fig. 16a and maximizing the variable moment of inertia of the plates, to reduce the bending effect.

Similarly to the procedure applied in section 4.1, a simplified model (see Fig. 16b) is developed to determine the deflection of the plate.
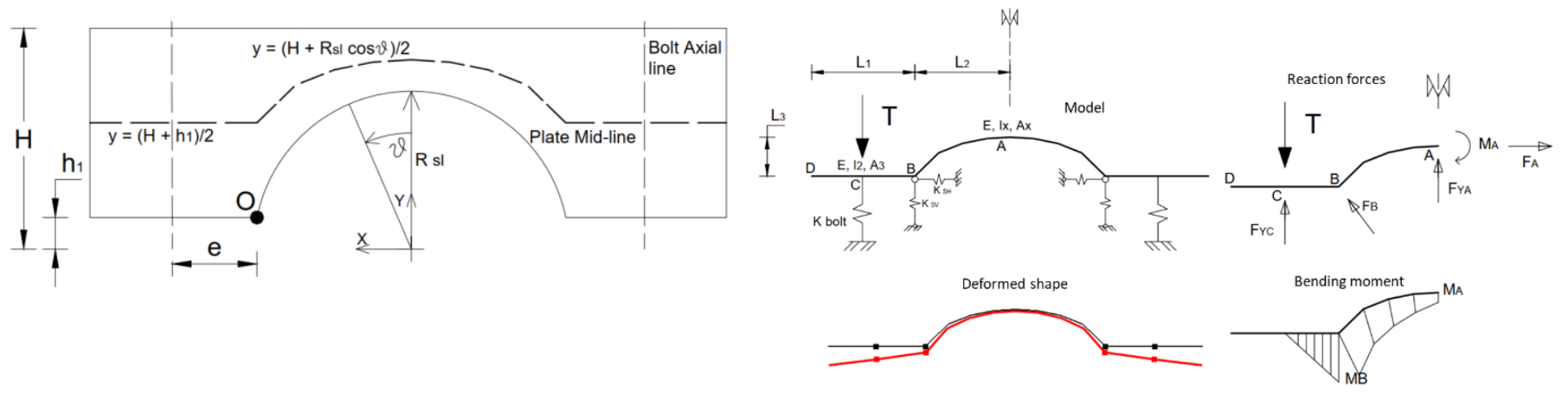

Fig. 16. Refined prototype: a) Cross-section of clamping plate, b) numerical model

The results highlight that for the same values of vertical load, the deformation due to bending is reduced compared to the SteSq model, as the moment of inertia of the curved portion increases with the bending moment distribution. Moreover, the plate transmits no bending moment to the bolts, hence avoiding yielding of the bolts head.

This is confirmed by observing the resultant deformation across the cross section of a 3D numerical model of the new prototype produced in Abaqus, where transversal bending for the same level of applied bolt preload, is negligible with respect to the results for the SteSq device presented in Fig. 14. The model also proves that the new geometry results in larger overall contact area with smaller patches of localized stresses, an order of magnitude smaller than the ones computed for device SteSq, (see Fig. 17b), implying that localized wear will be reduced.

The change in shape also allows to address other concerns about the impact of the device in historic buildings: the overall width of the device is reduced so that it can be housed within the cored hole for the passive bonded anchor, without need for further removal of historic authentic material. The size of the bolts is reduced, and their number increased to deliver the same value of $F_{\text {perp (N). }}$.
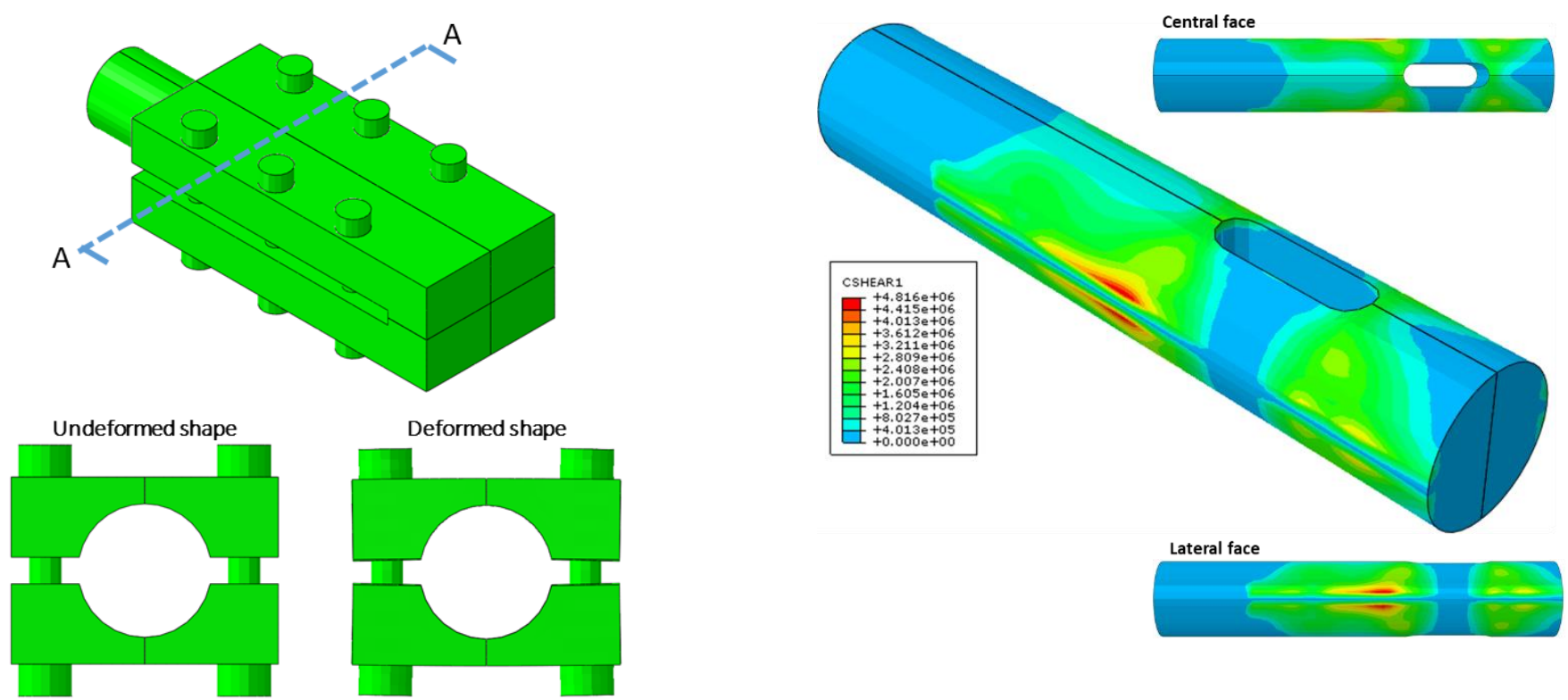

Fig. 17. Results of numerical analysis: a) deformed shape following the tightening of the bolt for a value of $10.7 \mathrm{KN}$, b) distribution of shear stresses over the sliding element.

However, the issue of abrasion and wear of the surfaces, is only partially resolved with the change in shape, as it also depends on the nature of the materials involved in the friction-controlled motion as already discussed in section 3 . Among the different materials that can be used for friction damping, in civil engineering application, the sliding of brass on steel exhibits a lower friction coefficient $(\mu=0.2)$, but also more stable force-displacement loops with lower material degradation than steel on steel. Alternatively, plastic-based materials, such as Teflon, are also commonly employed in wall-type dampers [52] and isolation systems [53] in seismic retrofitting design. They produce stable force-displacement loops with low material degradation, but large values of applied perpendicular pressure can affect the friction force, as 
it reduces with increasing normal pressure. Aiming to achieve low cyclic degradation, stable friction coefficient and effective damping variation within the acceptable thresholds, the new prototypes with the geometry shown in Fig. 18, are manufactured using three materials as frictional interfaces, namely steel, brass, and Fluorinated Ethylene Propylene (FEP), a plastic material known for its capacity to resist high temperatures and chemical agents.

a)

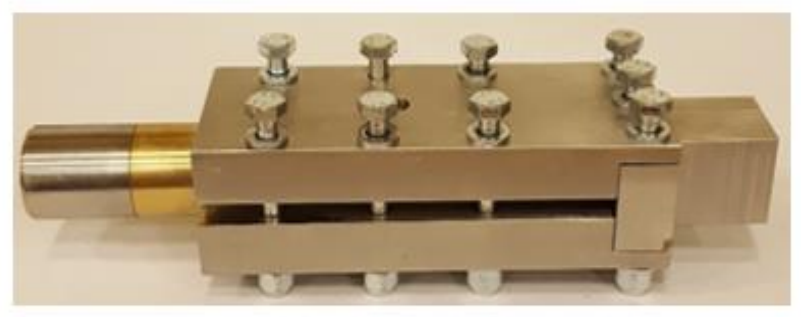

b)

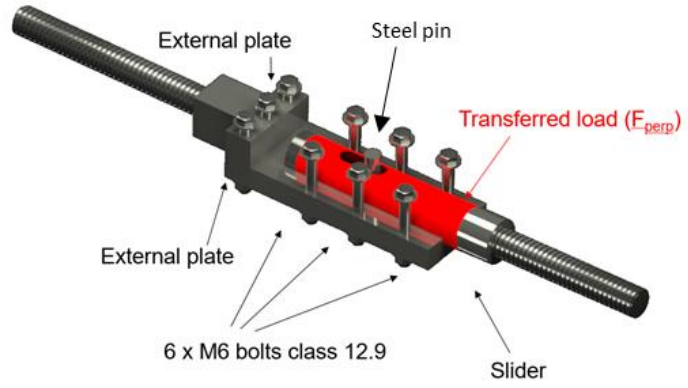

Fig. 18. Refined prototype (BraCyl), b) device's components and functioning.

The prototype's name, types of material in contact, nominal friction coefficient of the materials in contact and modulus of elasticity of the weaker material as reported by manufactures, are shown in Table 2 for each specimen.

Table 2. Properties of revised prototypes

\begin{tabular}{|c|c|c|c|}
\cline { 2 - 4 } Prototype Name & Type of Contact & $\mu(\mathrm{N})$ & $\mathrm{E}[\mathrm{Gpa}]$ \\
\hline SteCyl & Steel on Steel & 0.3 & 210 \\
\hline BraCyl & Brass on Steel & 0.2 & 97 \\
\hline FEPCYI & FEP on Steel & 0.15 & 0.7 \\
\hline
\end{tabular}

\section{Short-term assessment of revised prototypes}

Following the methodology (Fig. 3), the specimens are tested for short-term stability. The value of $k_{m}$ being affected by the shape of the bodies in contact and the bolts arrangement, the first test activity is repeated. Fig. 19a shows the values corresponding to the new prototype's geometry which mounts class 12.9 steel bolts with $6 \mathrm{~mm}$ in diameter.

The linear regression provides a value of $k_{m}=0.37$. While the increase in $k_{m}$ with respect to the SteSq prototype is only $6 \%$, the improved geometry shows two main advantages. Firstly, the relationship between torque and bolt preload remain linear for larger values of $\mathrm{M}$, confirming that no loss in bolt preload occurs due to local plasticity. Secondly, for equal values of torque, the bolt preload is $25 \%$ larger meaning that a larger perpendicular load can be achieved, as shown in Fig. 19a. The ultimate torque is set to $36.5 \mathrm{Nm}$, as computed from Eq. 4, with $d=6 \mathrm{~mm}, k_{m}=0.37, f_{u b}=1200 \mathrm{MPa}$ and $A s=20.1 \mathrm{~mm}^{2}$. However, for $M=35 \mathrm{Nm}$ the bolts displayed brittle failure in most of the cases. This is due to the bending moment produced during tightening, which becomes relevant as the bolt preload approaches the proof load $\left(F_{p}=16.8\right.$ $K N)$. The additional tensile stress associated to the bending deformation causes the yielding of the bolt along the bolt's shank, which then fails in a brittle manner, due to the lower ductility of 12.9 class bolts compared to 8.8 bolts. To avoid this effect, the ultimate value of reference torque is set to $30 \mathrm{Nm}$. 
a)

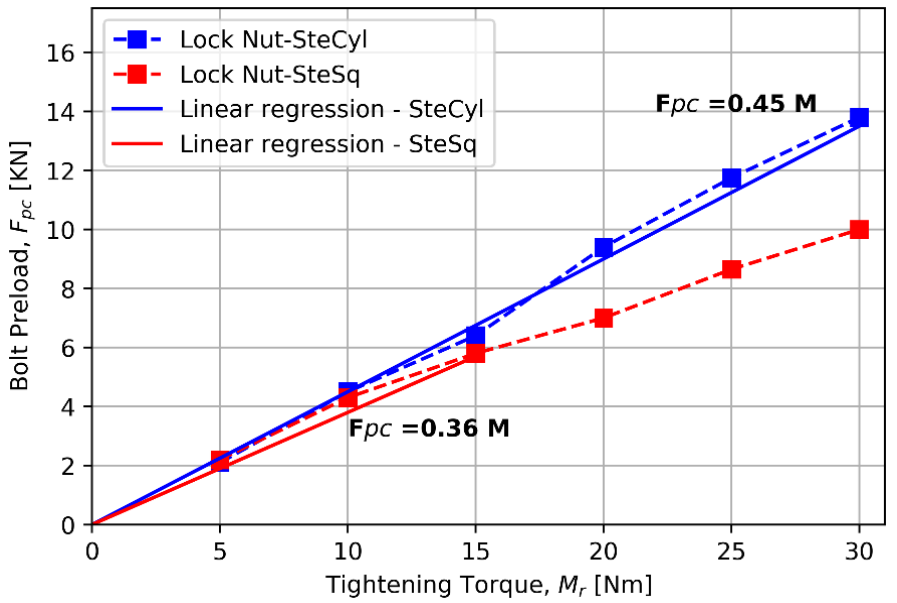

b)

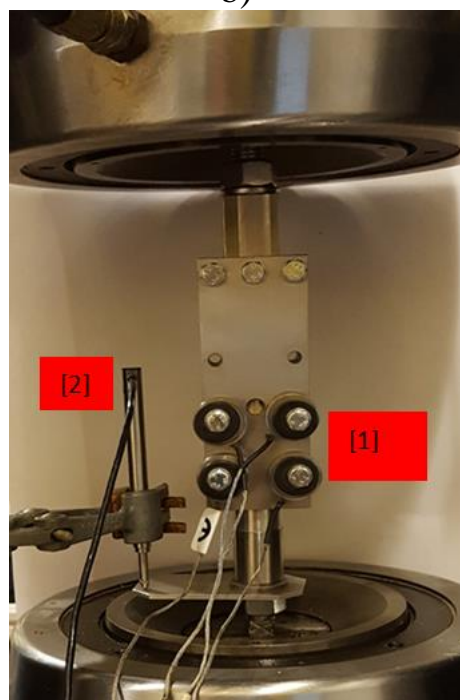

Fig. 19: a) Correlation between bolt pretension (Fpc) and applied bolt torque (M), b) Testing apparatus [1] Load cells, [2] LVDT.

Following the updated design and calibration of the bolt torque, the three devices are subjected to cyclic loading to assess the reliability of the different friction interfaces. The $\mathrm{SteCyl}$ and $\mathrm{BraCyl}$ devices are tightened with 4 bolts at 15 $\mathrm{Nm}$ torque, while the FEPCyl is tightened with 6 bolts at $20 \mathrm{Nm}$ torque, to compensate for its smaller nominal friction coefficient. Four pressure cells are installed below the head of four bolts to record the variation of the pretension transmitted throughout the testing activity. The testing apparatus also comprises an LVDT positioned on the slider to measure the relative motion of the slider with respect to the clamping plates as shown in Fig. 19b. The tests are carried out imposing 20 sinusoidal displacement cycles with peak stroke $d= \pm 6 \mathrm{~mm}$ and loading frequency $f=0.1 \mathrm{~Hz}$.

Fig. 20 shows the results for the three variants of the second prototype in terms of load-displacement loops. The figure reports also the number of tightened bolts, the nominal bolt preload, the design force and the wear condition of the sliders after 20 cycles of load.
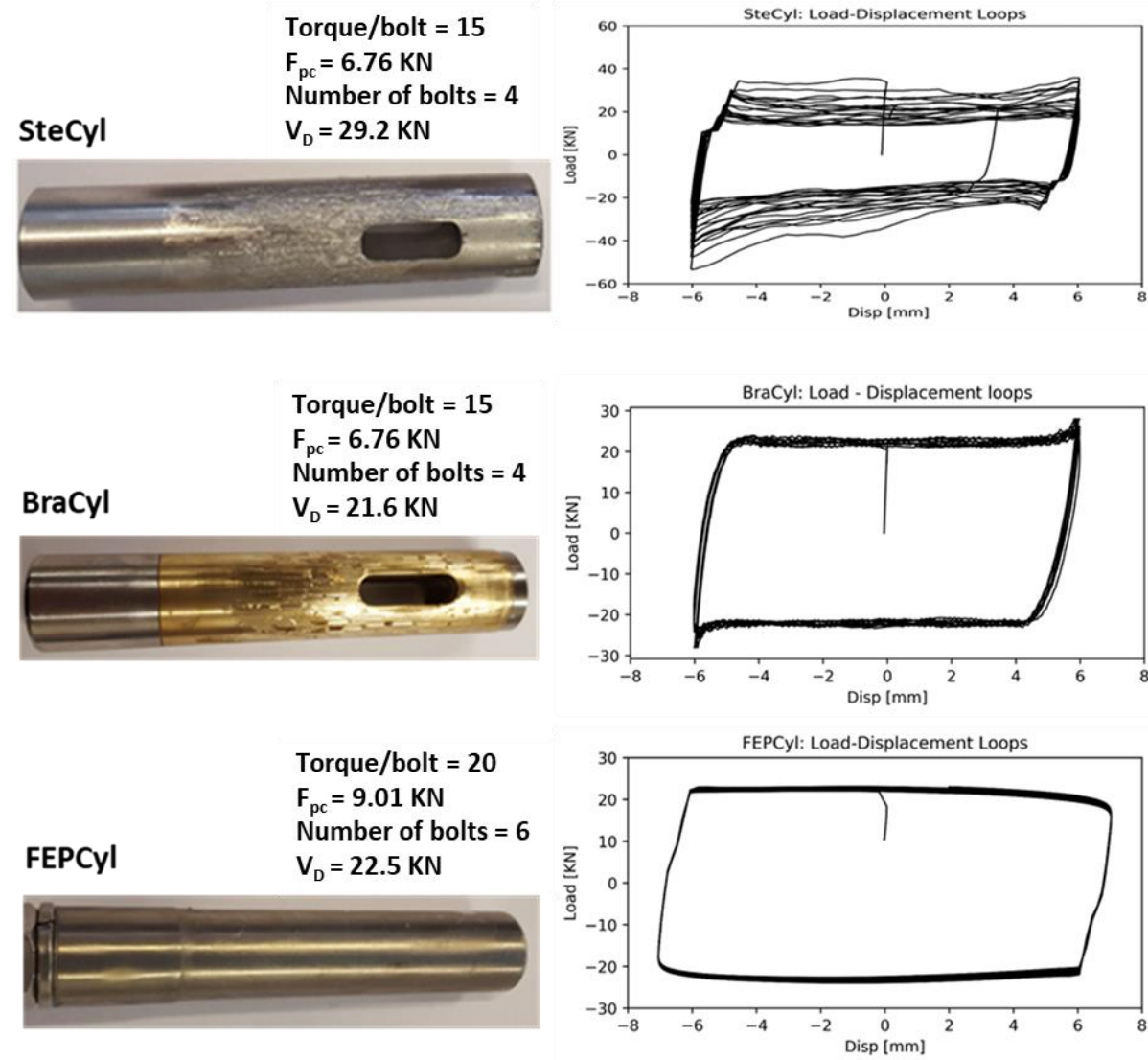

Fig. 20. Comparison between the three model for revised prototype: wear conditions, load-displacement loops, and loading/geometry conditions. 
Table 3 reports the parameters recorded during the testing activity for each prototype. The values obtained for the SteSq at $\mathrm{M}=15 \mathrm{Nm}$ are also included, for comparison.

Table 3. Summary of the test's results

\begin{tabular}{|c|c|c|c|c|c|}
\hline & & \multicolumn{4}{|c|}{ Prototype } \\
\hline \multicolumn{2}{|c|}{ Variables } & SteSq & SteCyl & BraCyl & FEPCyl \\
\hline $\mathrm{Mr}_{\mathrm{r}}$ & {$[\mathrm{Nm}]$} & 15 & 15 & 15 & 20 \\
\hline$F_{p c}$ & {$[\mathrm{KN}]$} & 5.36 & 6.76 & 6.76 & 9.01 \\
\hline$F_{\text {perp (N) }}$ & {$[\mathrm{KN}]$} & 21.4 & 27.03 & 27.03 & 54.05 \\
\hline$F_{\text {perp } 0(M)}$ & {$[\mathrm{KN}]$} & 20 & 26.5 & 28 & 53 \\
\hline$\beta$ & [\%] & $93 \%$ & $98 \%$ & $104 \%$ & $98 \%$ \\
\hline$\mu_{0(\mathrm{M})}$ & {$[-]$} & 0.32 & 0.16 & 0.19 & 0.103 \\
\hline$\mu_{\max (M)}$ & {$[-]$} & 0.392 & 0.35 & 0.22 & 0.105 \\
\hline$\mu_{D}$ & {$[-]$} & 0.36 & 0.27 & 0.21 & 0.104 \\
\hline $\max \left(\Delta \mu_{(M)}\right)$ & {$[-]$} & $11 \%$ & $41 \%$ & $10 \%$ & $1 \%$ \\
\hline$V_{D}$ & {$[\mathrm{KN}]$} & 30.9 & 29.2 & 22.7 & 22.49 \\
\hline$F_{s l, 3}$ & {$[\mathrm{KN}]$} & 26.3 & 29.17 & 23.06 & 23.62 \\
\hline $\mathrm{F}_{\mathrm{sl}(\mathrm{mim})}$ & {$[\mathrm{KN}]$} & 24.40 & 18 & 20.1 & 21 \\
\hline$F_{s l}(\max )$ & {$[\mathrm{KN}]$} & 31.1 & 51.5 & 24.4 & 23.62 \\
\hline$\xi_{\text {effb }, 3}$ & {$[-]$} & 0.52 & 0.6 & 0.68 & 0.63 \\
\hline $\max \left(\Delta \xi_{\text {effb })}\right)$ & [\%] & $18.3 \%$ & $76 \%$ & $10.4 \%$ & $4.3 \%$ \\
\hline$S_{w}$ & {$[\mathrm{~mm}]$} & 1.5 & 1.00 & 0.50 & 0.3 \\
\hline
\end{tabular}

Specimen SteCyl shows a dramatic increase in slip load between the first and the last cycles and severe signs of wear are noticeable both on the slider and the plates. The signs of abrasion are spread over all the surface of the cylindrical slider, rather than concentrated only on the edges, proving that the cylindrical shape determines a better use of the available contact area. On the other hand, the use of a larger contact area determines larger quantity of material dust and widely spread signs of degradation, which increase the occurrences of interlocking and adherence between the parts, thus increasing the resistance to sliding. As a result, for the SteCyl prototype $\Delta \xi_{e f f b}$ and $\Delta \mu_{(\mathrm{M})}$ reach a maximum of $76 \%$ and $40 \%$ respectively and a severe wear rate is registered. In the case of the BraCyl prototype, the application of the brass sleeve improves the stability of the load-displacement loops and the maximum $\Delta \xi_{\text {eff }}$ is about $10 \%$, thus within the threshold prescribed by EN 15129. Similarly, to the SteCyl and SteSq, the slippage force measured for the BraCyl increases with the number of cycles, but the hardening behaviour is less marked. For the FEPCyl specimen, the use of the FEP tube around the slider stabilizes even further the hysteresis loops and the $\Delta \xi_{\text {effb }}$ is no greater than $7.2 \%$. The use of a propylene rather than a metallic material as friction interface has the additional effect of removing the load peaks noticeable when the slider inverts its motion, passing from a state of dynamic to a state of static friction.

For the loading conditions reported in Table 3, the variations of the $\mu_{(\mathrm{M})}$ during the test are summarized in Fig. 21 for the three cylindrical specimens and the SteSq at $\mathrm{M}=15 \mathrm{Nm}$. The design values of friction coefficient and activation load are also presented in Table 3. Both brass and FEP materials proved to be efficient friction interfaces as they present a stable friction coefficient throughout the test. The FEPCyl prototype displays mild softening behaviour after the third cycle, registering a decrement of $1 \%$ in the friction coefficient with respect to the initial value, as expected for synthetic polymers subjected to cyclic sliding motion [36]. 

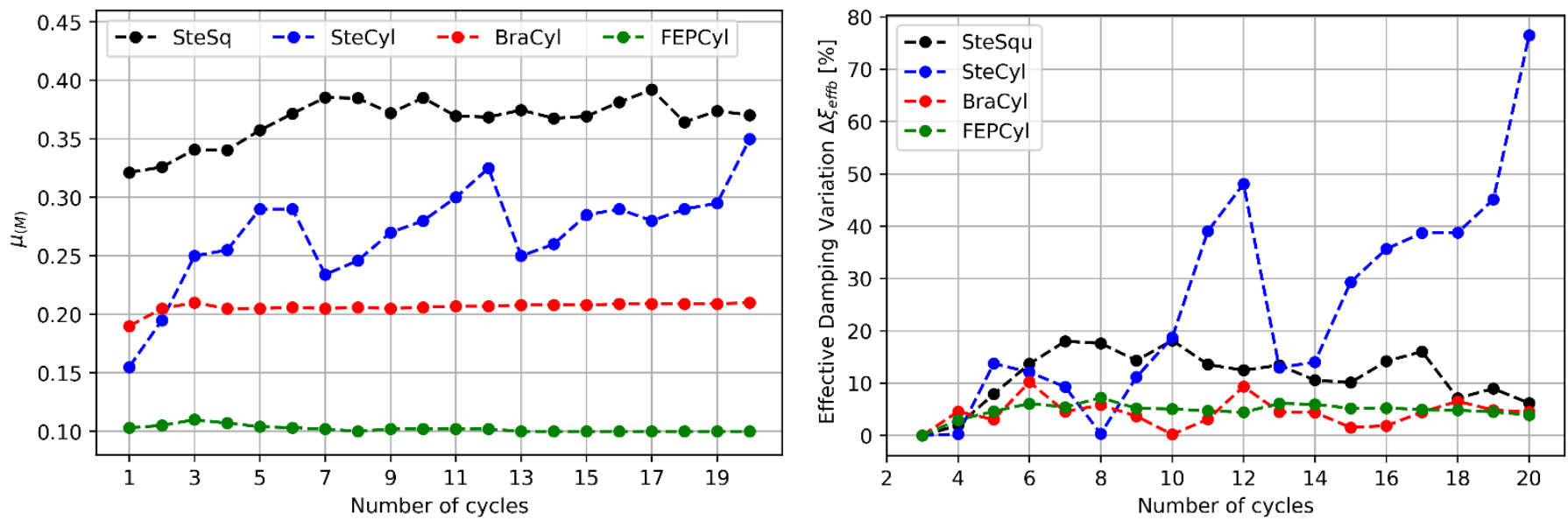

Fig. 21. Variation of the friction coefficient $\mu_{(\mathrm{M})}$ for 20 cycles of load. Comparison between the prototypes of the revised device and SteSq at $\mathrm{M}=15 \mathrm{Nm}$.

Given the abrasion depth and the computed max $\left(\Delta \mu_{(\mathrm{M})}\right)$ (reported in Table 3), the $S_{\mathrm{w}}$ of BraCyl and FEPCyl is "mild with low wear rate". This result is expected as both the brass and FEP have lower elastic modulus $E$ compared to stainless steel.

Eq. 13 provides reasonable results for metals, but in case of polymeric-based materials, such as FEP, other factors such as contact pressure (P), and the sliding speed (v) might affect the wear rate and the coefficient of friction [54]. Therefore, to conclude the short-term assessment of the FEPCyl prototype, the maximum variations of $\mu_{\mathrm{M}}$ and $\xi_{\text {effb }}$ are investigated for values of bearing pressure and sliding speed compatible with seismic engineering applications.

The displacement history of Eq. 12 is imposed at frequency values of 0.5 and $2 \mathrm{~Hz}$ and exploiting the full sliding amplitude of the slider, $d= \pm 10 \mathrm{~mm}$, as recommended by EN 15129. By varying the frequency of the displacement wave form while keeping the amplitude fix, the sliding velocities of $20 \mathrm{~mm} / \mathrm{s}$ and $80 \mathrm{~mm} / \mathrm{s}$ are obtained. The device is tested for two values of reference torque, 20 and $25 \mathrm{Nm}$, and the corresponding load-displacement loops are shown in Fig. 22.

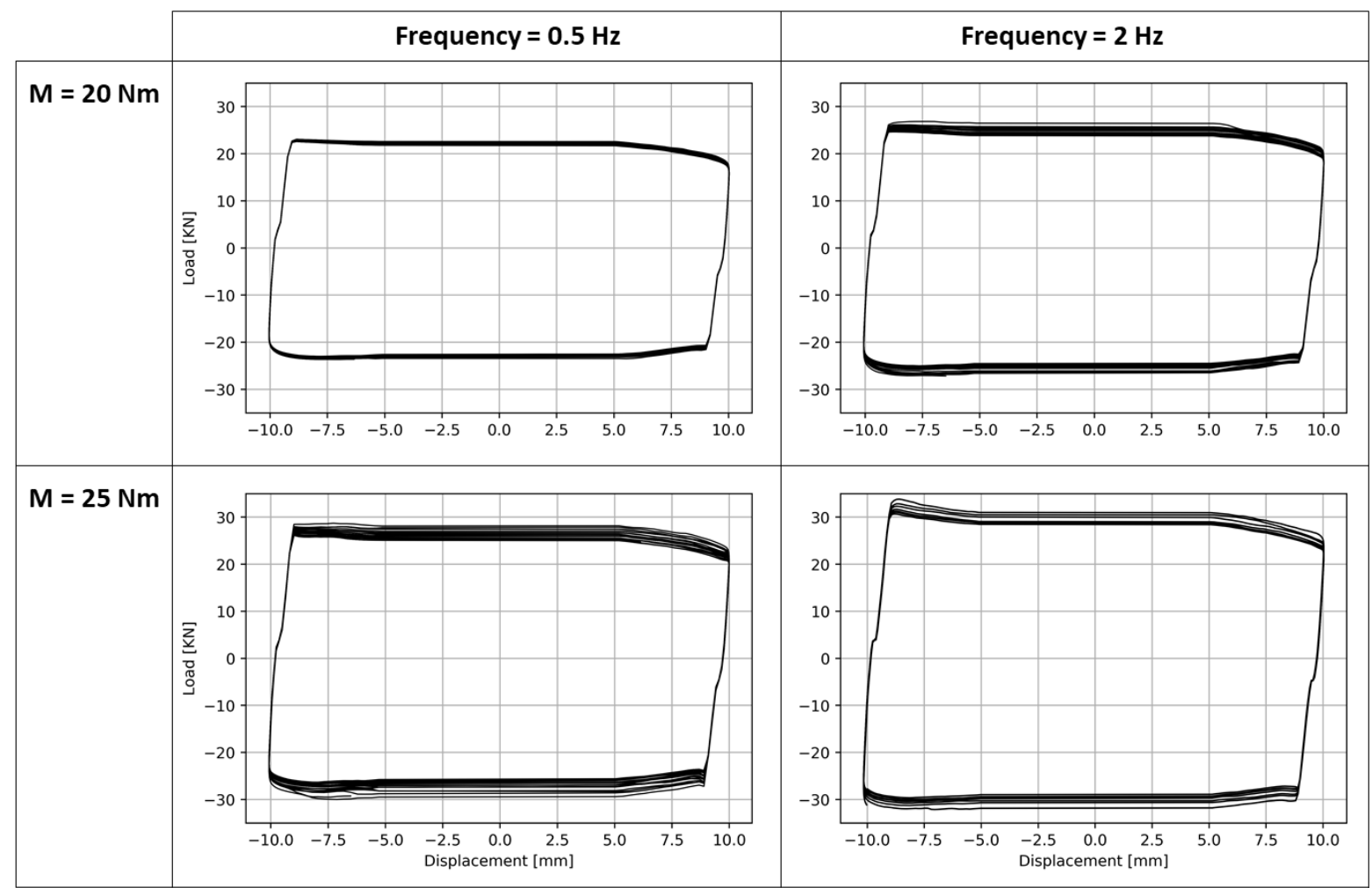

Fig. 22. Load-displacement loops of FEPCyl at $f=0.5 \mathrm{~Hz}$ and $2 \mathrm{~Hz}$, and $\mathrm{M}=20 \mathrm{Nm}$ and $25 \mathrm{Nm}$

For the same value of torque to the bolts, larger values of slippage force are registered for the higher value of frequency. The friction coefficient was computed for each cycle according to Eq. 13, and the variation of $\mu_{M}$ is shown in Fig. 23 a. 
From the graph, it can be seen that $\mu_{M}$ increases with increasing velocities and that follows a softening behaviour during testing. Conversely, the friction is inversely proportional to the pressure acting on the slider. Fig. 23b shows the evident effect of velocity and pressure on the sliding coefficient of friction for FEP. The mean values of friction coefficients over 20 cycles are reported, along with the value of $\mu_{\mathrm{M}}$ at $f=0.1 \mathrm{~Hz}$ (sliding velocity $=4 \mathrm{~m} / \mathrm{s}$ ), as obtained from previous tests. The value of the coefficient of friction increases with an increase in the sliding velocity up to a threshold beyond which it remains constant. This value of velocity is between 100 and $200 \mathrm{~mm} / \mathrm{s}$. This experimental evidence is in agreement with the results found by other researchers which tested the correlation between values of friction coefficients, sliding velocity and applied pressure for plastic-based materials [55,56].

a)

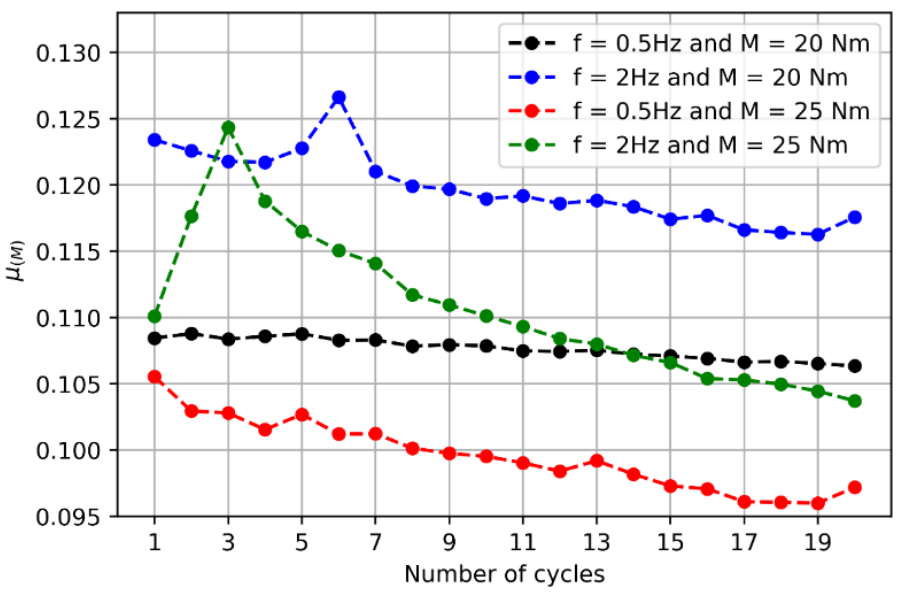

b)

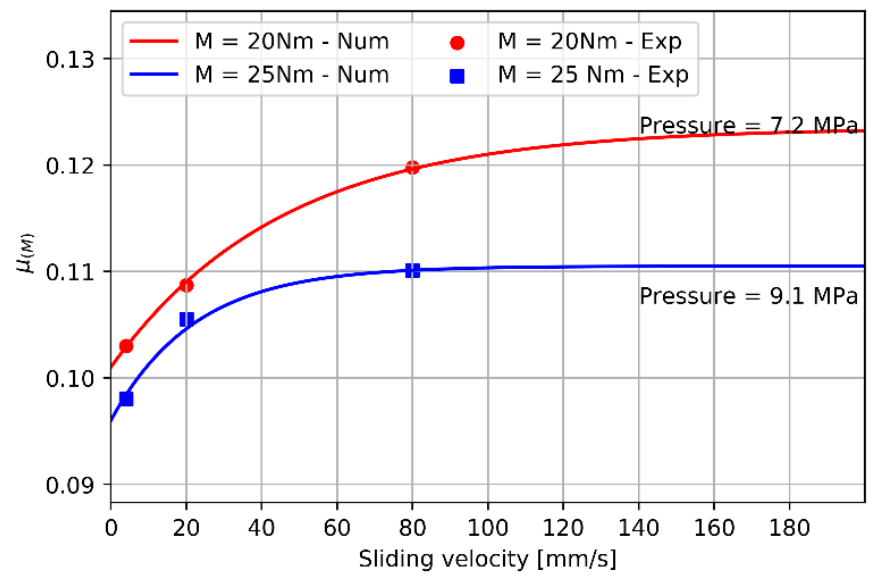

Fig. 23. a) Variability of $\mu_{M}$ over 20 cycles of load for $f=0.5$ and $2 \mathrm{~Hz}$, and $\mathrm{M}=20$ and $25 \mathrm{Nm}$. b) Variation of Sliding Coefficient of Friction with Velocity and Pressure

As proposed by [56], the coefficient of friction at sliding velocity $V$ may be approximated by the following equation:

$$
\mu_{s}=\mu_{\max }-D_{f} \exp (-a V)
$$

in which $\mu_{\max }$ is the coefficient of friction at large velocity of sliding (after leveling off), $D_{f}$ is the difference between $\mu_{\max }$ and the sliding value at very low velocity, $a$ is a constant for given bearing pressure.

Table 4 presents values of $\mu_{\max }, D_{f}$, and $a$ for various pressure conditions that resulted in the solid-line curves in Fig. 23b. It is apparent that Eq.18 reproduces the results of experiments with good accuracy and that it can be used for a more accurate description of the coefficient of friction for the FEP interface. The maximum variation of $\mu_{(\mathrm{M})}$ is within the limit of $15 \%$ and $\max \left(\Delta \xi_{\text {eff }}\right)$ is below $10 \%$ for all cases, meaning that the FEP interface is able to deliver a stable behaviour under different conditions of pressure and sliding velocity. For practical uses, the value of $\mu_{(D)}=0.11$ is recommended to compute the design force of the FEPCyl device.

Table 4. Values of $\mu$ recorded at different sliding velocities and numerical parameters to compute $\mu_{s}$

\begin{tabular}{|c|c|c|c|c|c|c|c|c|c|}
\hline $\begin{array}{c}\text { Torque, } \\
\mathrm{M} \\
{[\mathrm{Nm}]}\end{array}$ & $\begin{array}{c}\text { Pressure, } \mathrm{P} \\
{[\mathrm{MPa}]}\end{array}$ & 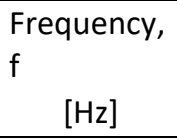 & $\begin{array}{l}\text { Sliding Velocity, v } \\
{[\mathrm{mm} / \mathrm{s}]}\end{array}$ & $\begin{array}{c}\mu(D) \\
{[-]}\end{array}$ & $\begin{array}{c}\max (\Delta \mu \\
(\mathrm{M})) \\
{[-]}\end{array}$ & $\begin{array}{c}\left.\max \left(\Delta \xi_{\mathrm{effb}}\right)\right) \\
{[-]}\end{array}$ & $\begin{array}{c}\mu_{\max } \\
{[-]}\end{array}$ & $\begin{array}{l}D_{f} \\
{[-]}\end{array}$ & $\begin{array}{c}a \\
{[-]}\end{array}$ \\
\hline \multirow{3}{*}{20} & \multirow{3}{*}{7.3} & 0.1 & 4 & 0.103 & $1 \%$ & $4.30 \%$ & \multirow{3}{*}{0.123} & \multirow{3}{*}{0.026} & \multirow{3}{*}{0.022} \\
\hline & & 0.5 & 20 & 0.109 & $1.20 \%$ & $1.90 \%$ & & & \\
\hline & & 2 & 80 & 0.119 & $2 \%$ & $5 \%$ & & & \\
\hline \multirow{3}{*}{25} & \multirow{3}{*}{9.1} & 0.1 & 4 & 0.094 & $2 \%$ & $5 \%$ & \multirow{3}{*}{0.11} & \multirow{3}{*}{0.014} & \multirow{3}{*}{0.045} \\
\hline & & 0.5 & 20 & 0.1 & $2.40 \%$ & $6.60 \%$ & & & \\
\hline & & 2 & 80 & 0.11 & $6 \%$ & $9.0 \%$ & & & \\
\hline
\end{tabular}

\section{Long-term stability - Laboratory tests on single device}

Both specimens BraCyl and FEPCyl present values of $\Delta \xi_{e f f b}$ within the maximum variation allowed by EN 15129 [13], meaning that no further improvement of the design is required and that both device have a stable and reliable short-term 
behaviour. Therefore, in line with the testing methodology proposed, the long-term stability and reliability of the dissipative devices is experimentally investigated. Along with BraCyl and FEPCyl, the SteSq prototype is tested for the long-term behaviour. Even if it presents a maximum value of $\Delta \xi_{\text {eff }}$ above the limit of $10 \%$, the variation is less pronounced than the SteCyl and hence considered in preference to the latter for the environmental aging.

According to EN 15129, the seismic device shall remain operational also for the range of environmental conditions expected during its projected service life. For the testing activity, the expected environmental conditions are obtained by considering the average variation of the temperature and humidity in an earthquake-prone city over a period of 50 years. L'Aquila, a city in the centre of Italy that suffered from a 6.3-magnitude earthquake in 2009 [23], is taken as reference for the temperature (T) and relative humidity (RH) ranges, which vary from $-1^{\circ} \mathrm{C}$ to $27^{\circ} \mathrm{C}$ and from $50 \%$ to $80 \%$ respectively within a period of one year [57]. The first 5 cycles are shown in Fig. 24b: each cycle is completed in one day and corresponds to the variation of temperature and humidity of one year. These values of $\mathrm{T}$ and RH represent the expected in-wall conditions of masonry walls, as they are in good agreement with values recorded by long term field monitoring of historical buildings [42,43].

a)

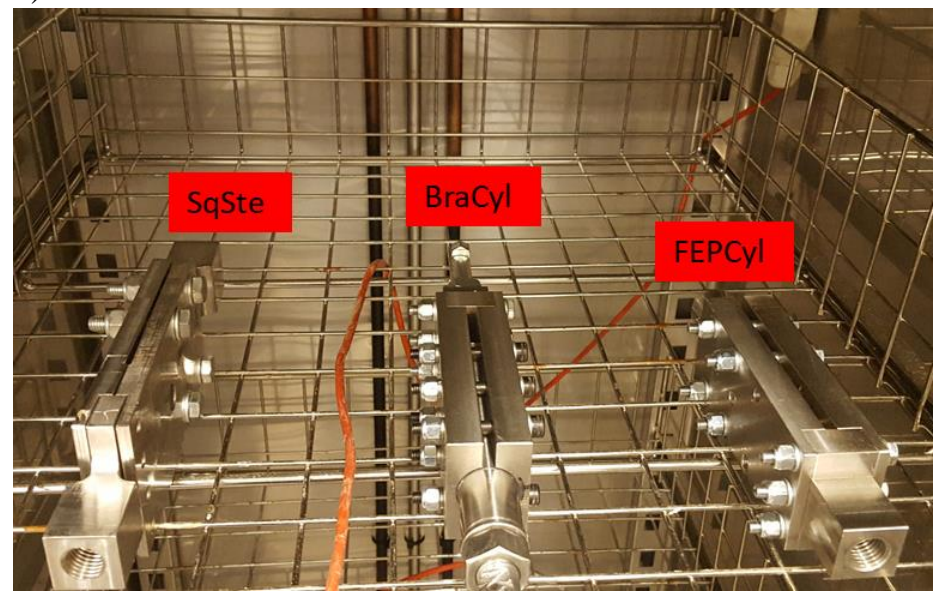

b)

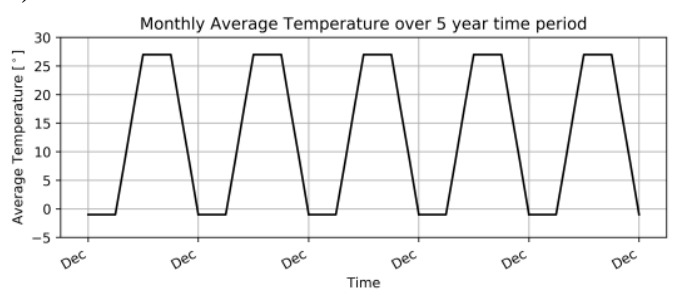

c)

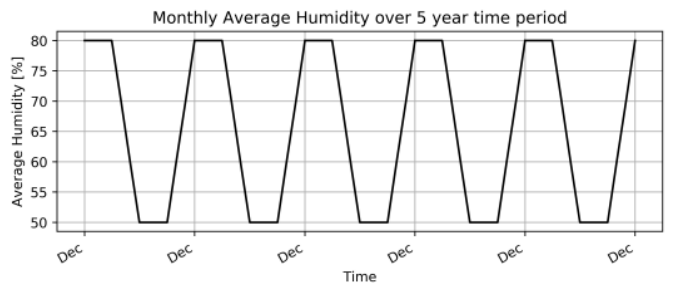

Fig. 24: a) Devices positioned in the thermostatic cabinet, cycles of temperature (b) and relative humidity (c)

The devices are pre-tuned to the same values of reference torque imposed in the short-term analysis and then are placed in a thermostatic cabinet for 14 days, subjecting them to temperature and humidity cycles. Once the thermal and environmental conditioning is over, the "aged" devices are individually tested in displacement-control mode for 20 cycles of load $\left(\mathrm{d}_{0}= \pm 5 \mathrm{~mm}, \mathrm{f}_{0}=0.1 \mathrm{~Hz}\right)$ and the results compared with those conducted on the pristine devices (referred to as "unaged" devices). The devices are deemed to have a reliable and stable long-term behaviour if the variation in effective damping does not exceed a limit value of $15 \%$. The initial preloading conditions and the values of the main variables are reported in Table 5.

Table 5. summary of results of loading test on aged devices

\begin{tabular}{c|c|c|c|c|c|c|c|c|c|c|}
\hline Prototype & $\begin{array}{c}\mathrm{M}_{\mathrm{r}} \\
{[\mathrm{Nm}]}\end{array}$ & $\begin{array}{c}\mathrm{F}_{\mathrm{pc}} \\
{[\mathrm{KN}]}\end{array}$ & $\begin{array}{c}\mathrm{F}_{\text {perp }(\mathrm{N})} \\
{[\mathrm{KN}]}\end{array}$ & $\begin{array}{c}\mu_{\mathrm{D}} \\
{[-]}\end{array}$ & $\begin{array}{c}\mathrm{V}_{\mathrm{D}} \\
{[\mathrm{KN}]}\end{array}$ & $\begin{array}{c}\mathrm{F}_{\mathrm{sl}, 3} \\
{[\mathrm{KN}]}\end{array}$ & $\begin{array}{c}\mathrm{F}_{\mathrm{sl}}(\mathrm{min}) \\
{[\mathrm{KN}]}\end{array}$ & $\begin{array}{c}\mathrm{F}_{\mathrm{sl}}(\max ) \\
{[\mathrm{KN}]}\end{array}$ & $\begin{array}{c}\xi_{\text {effb,3 }} \\
{[-]}\end{array}$ & $\begin{array}{c}\max \left(\Delta \xi_{\text {effb_aged }}\right) \\
{[\%]}\end{array}$ \\
\hline SteSq & 15 & 5.36 & 21.4 & 0.37 & 31.7 & 28.5 & 27.10 & 43 & 0.57 & $50.9 \%$ \\
\hline BraCyl & 15 & 7.14 & 28.57 & 0.20 & 22.9 & 22.8 & 21.5 & 25.2 & 0.64 & $10.5 \%$ \\
\hline FEPCyl & 20 & 9.52 & 57.14 & 0.110 & 25.14 & 25.1 & 23.5 & 25.6 & 0.64 & $6.4 \%$ \\
\hline
\end{tabular}

The weathering procedures proved useful into exploring the development of corrosion in the device's metal components. Looking at the results reported in Fig. 25, the load displacement loops for the SteSq prototype show that the performances of this device are greatly affected by the imposed aging conditions: the slip load is greater than the design force $V_{D}$ and the device displays a hardening behaviour with the progressing of the test. 


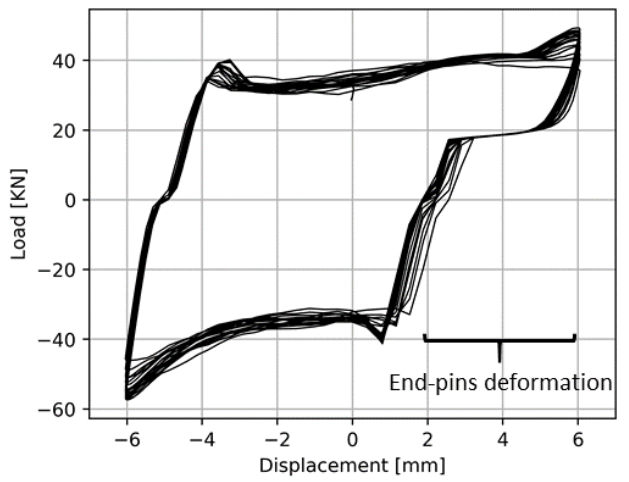

a)

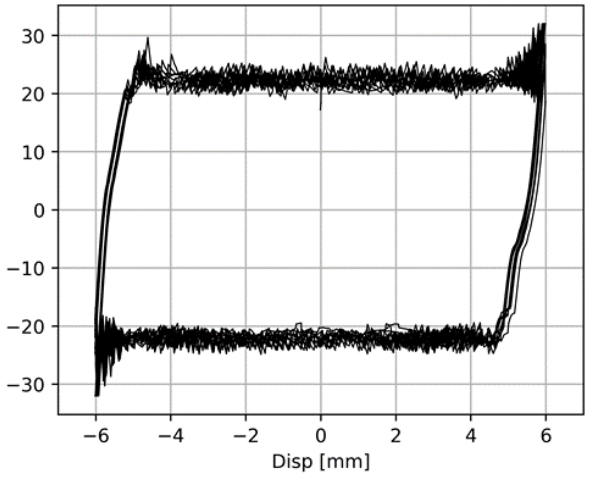

b)

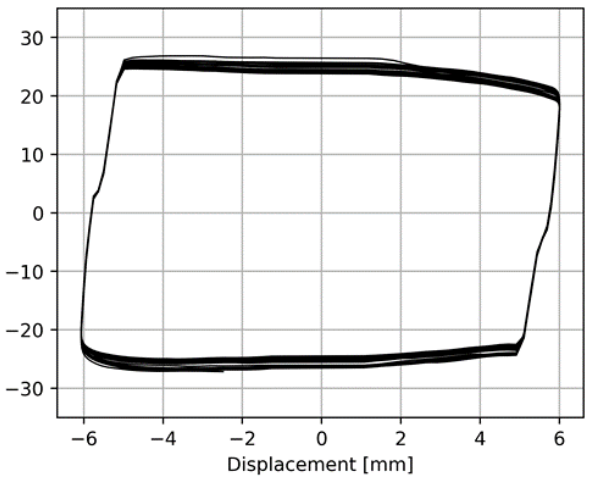

c)

Fig. 25:. Results of load cycle test on aged devices: a) SteSq, b) BraCyl, c) FEPCyl

The central dowel pin used to control the slider's motion and the end-pins assembling the plates together showed severe signs of corrosion (Fig. 26b). Dowel pins are used to keep device's components alignment purposes but had not been treated by the manufacturer to resist aggressive weathering conditions. This causes additional friction between the slider and the central dowel pin thus increasing the slippage load. In particular, the slippage force is almost twice the design slippage load in the stick-slip phase. Moreover, the corrosion weakened the mechanical strength of the end-pins which deform in shear when the motion reverses and the slider is pushed into the assembly. As a result, a relative motion of $2.4 \mathrm{~mm}$ is recorded, as visible in Fig. 25a. To avoid this negative effect in the second-generation prototypes and to increase the stiffness of the fix part of the device the pins were replaced with high strength bolts in the "design refinement" task.

Regarding the BraCyl prototype, the fastener that holds in place the brass tube of the BraCyl device failed during the first cycle of load and the test was suspended. A visual inspection of the device highlighted that the presence of two materials with different electrical potential in contact had caused the development of galvanic corrosion, as shown in Fig. 26a. A class 12.9 zinc-coated fastener was adopted to replace the faulted one and the device was placed again in the thermostatic cabinet for 14 days before repeating the loading test. Because zinc is a lesser noble metal than iron steel, the zinc will corrode first, protecting the steel of the fastener against rusting until all zinc has dissolved [41].

The load-displacement loops reported in Fig. 25b highlight that the BraCyl prototype has a stable behaviour during sliding with a slip load comparable to the one obtained for a fresh device. Nonetheless, the static load recorded when the slider inverts its motion is about $40 \%$ greater than the load recorded during motion, while the stick-slip phase was less evident (30\% increase) for the unaged BraCyl prototype.

The FEPCyl device (Fig. 25c) displays the most stable behavior with a slightly larger maximum variation of damping $\Delta \xi_{\text {effb (aged })}=6.4 \%$ with respect to the unaged prototype $\left(\Delta \xi_{e f f b}=4.3 \%\right)$. As for the unaged prototype, the FEP tube eliminates the end-run spikes, resulting in the most reliable behaviour among the three prototypes.

a)

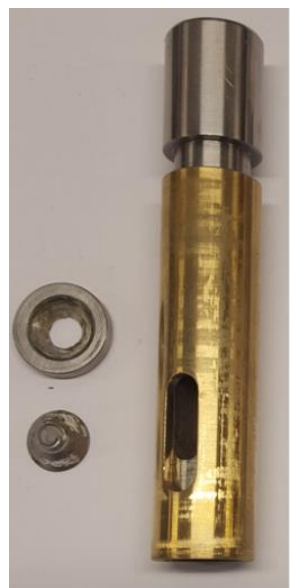

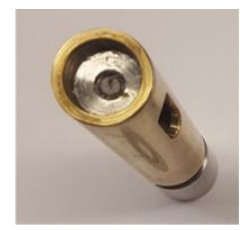

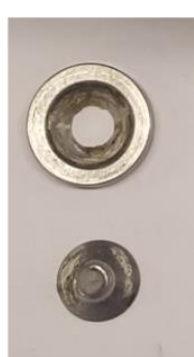

b)

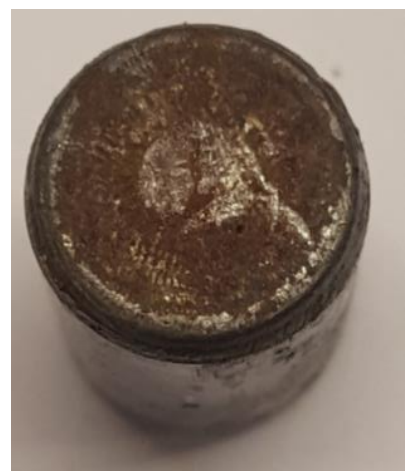

Fig. 26: a) Signs of galvanic corrosion of the BraCyl fastener b) corrosion of the central dowel pin for the SteSq prototype. 


\section{Summary of tests results and conclusions}

The study presents a methodology for the assessment, design, and refinement of friction-based dissipative devices. The methodology comprises three main activities: two experimental activities explore the short-term and long-term stability of the device under cyclic loading, comparing the test results with the performance requirements recommended by the appropriate standard. The third activity investigates, by advanced 3D numerical simulations, alternative design solutions able to reduce the maximum variation of the prototype's damping, $\max \left(\Delta \xi_{e f f b}\right)$, down to $10 \%$, as recommended by the EN 15129 and to ensure long term stability by design. The $\max \left(\Delta \xi_{e f f b}\right)$ has proven to be effective in describing the device performance in terms of both the variation of the slippage load in respect to the nominal design force $V_{D}$ for each cycle, and of the stability of the response within the 20 load cycles with respect to the reference third cycle.

The validity of the proposed methodology is first tested on the patented prototype of friction-based device, the SteSq, which is part of a dissipative anchoring system designed for the seismic strengthening of historical buildings. The results of the experimental activities highlighted that the performance of the SteSq device varies by $18 \%$ for the short-term test, beyond the limit set by EN 15129. This clearly outline a need for re-design.

The design by analysis leads to the implementation of a cylindrical slider, in place of the squared one, which proves to increase the contact area, and improve the clamping efficiency of the bolts. According to this result, three prototypes are produced and ad-hoc material, such as brass and FEP, are implemented as friction interfaces, to address the issue of high wear rates.

Compared to the SteSq, the second generation of prototypes reduces the deflection of the enclosing plate, thus achieving larger bolt preloading for equal values of torque. Under cyclic loading, the prototypes featuring the brass and FEP friction interfaces displayed $10 \%$ and $4 \%$ of variability in effective damping and less severe sign of wear, hence well within the EN 15129 performance requirement.

The procedure needed to compute the design force of the devices is obtained from the results of the experimental activities and draws on the basic formulas valid for bolted unions and dry friction. Nonetheless, the experimental results display that the formulation provided by EN 1090-2 for bolted connections overestimates the nominal perpendicular force, with an error of about $42 \%$ for the SteSq and of $28 \%$ for the cylindrical devices.

Therefore, two empirical values of $k_{m}$ are computed specifically for the devices' geometry achieving an accuracy of above $95 \%$ between the nominal and recorded values of perpendicular force. Then, the design force $V_{D}$ is computed in a straightforward way from Eq. 14 considering four friction surfaces $(n=4)$ and values of $\mu$ equal to $0.36,0.21$ and 0.11 for the SteSq, BraCyl and FEPCyl, respectively.

Additionally, the revised devices and the SteSq prototype are tested after being exposed to cycles of temperature and relative humidity for fourteen days in a thermostatic cabinet. This type of tests aims at evaluating the long-term stability of anti-seismic devices which is rarely investigated in literature, even though these devices often comprise different metals or high-tech materials that might be affected by aggressive environmental conditions. This lack of knowledge is partially due to the insufficient guidance of EN 15129: while stating that the effects of ageing should be evaluated by repeating the cyclic loading tests in different exposure conditions, it remains vague on the details, especially for displacement-dependant devices that do not carry vertical loads. Given this lack of guidance, the ranges for the environmental variables were derived from expected in-wall conditions from masonry walls in an earthquake-prone region in relatively extreme climatic conditions (hot summers, cold winters, high level of moisture).

The test's results highlight the weakest parts of the devices, which in some case failed due to low corrosion resistance. Moreover, it is clear that the aging conditions can affect the performance of the devices: the SteSq displayed and increment in max $\left(\Delta \xi_{\text {effb_aged }}\right)$ up to $50 \%$ while the increment was less marked for the BraCyl and FEPCyl which remained within the limits of EN. Given these results, further tests will be needed to investigate the resistance of the device to longer periods of weathering exposure and its corrosion resistance with respect to saline environment.

In conclusion, the methodology proved to be a valid tool for the complete assessment and validation of a friction-based dissipative device. Although the validity of the methodology was tested for a bolted slotted device, its use can be extended to other typologies of friction-based devices. For instance, devices consisting of an inner shaft shrink-fitted inside a second shaft would require a different test for the assessment of the imposed perpendicular load, but the performance parameters and procedural steps of the methodology remain valid.

Full scale experimental tests, currently under development, explore the behaviour of the cylindrical devices as connected with an anchor and the ductility provided by the dissipative systems when embedded in a corner connection of masonry walls. These tests will also provide better insight into the actual applicability and intrusiveness of the system. Design procedures of the dissipative anchoring system including the FEPCyl and BraCyl will be developed, calculating the load 
capacity of the systems components and of the grouted steel elements for different limit states, and hence obtaining a hierarchy of failure.

The achieved stability of $\mu_{(\mathrm{M})}$ and $\xi_{e f f b}$ will allow for a more accurate and simple design: the design force will better represent the actual slippage load, which must be smaller than the minimum capacity of the assembly to ensure the device's activation and the energy dissipation. Conversely, for the SteSq device $V_{D}$ was defined as a range of values for each perpendicular load[34], due to the high wear rate of the friction surfaces and the resulting large variability of $\mu_{(\mathrm{M})}$, thus hindering the reliability of the design. Given that prescriptions of codes with respect to unreinforced masonry connections are mainly qualitative [58], future work will also focus on providing practical guidelines for the implementation of the dissipative anchoring system to structural connections in historic buildings.

\section{Declaration of interests}

The authors declare that they have no known competing financial interests or personal relationships that could have appeared to influence the work reported in this paper.

\section{Funding declaration}

This research did not receive any specific grant from funding agencies in the public, commercial, or not-for-profit sectors.

\section{References}

[1] J.E. Martínez-Rueda, On the evolution of energy dissipation devices for seismic design, Earthq. Spectra. 18 (2002) 309-346. https://doi.org/10.1193/1.1494434.

[2] T.T. Soong, B.F. Spencer, Supplemental energy dissipation: State-of-the-art and state-of-the-practice, Eng. Struct. 24 (2002) 243-259. https://doi.org/10.1016/S0141-0296(01)00092-X.

[3] ICOMOS, Icomos Charter- Principles for the Analysis, Conservation and Structural Restoration of Architectural Heritage (2003), 2003.

[4] DPCM 2011., Linee guida per la valutazione e la riduzione del rischio sismico del patrimonio culturale con riferimento alle Norme Tecniche per le Costruzioni di cui al decreto del Ministero delle Infrastrutture e dei trasporti del 14 gennaio 2008 (09/02/2011), 1 (2011) 1-83.

[5] D. D'Ayala, Conservation Principles and Performance Based Strengthening of Heritage Buildings in Post-event Reconstruction, Geotech. Geol. Earthq. Eng. 34 (2014). https://doi.org/10.1007/978-3-319-07118-3_15.

[6] F. Arifovic, M.P. Neilsen, Strength of Anchors in Masonry, 2004.

[7] R. Muñoz, P.B. Lourenço, S. Moreira, Experimental results on mechanical behaviour of metal anchors in historic stone masonry, Constr. Build. Mater. 163 (2018) 643-655. https://doi.org/10.1016/j.conbuildmat.2017.12.090.

[8] S. Paganoni, D. D'Ayala, Testing and design procedure for corner connections of masonry heritage buildings strengthened by metallic grouted anchors, Eng. Struct. 70 (2014) 278-293. https://doi.org/10.1016/j.engstruct.2014.03.014.

[9] G. Maddaloni, M. Di Ludovico, A. Balsamo, A. Prota, Out-of-plane experimental behaviour of T-shaped full scale masonry wall strengthened with composite connections, Compos. Part B Eng. 93 (2016) 328-343. https://doi.org/10.1016/j.compositesb.2016.03.026.

[10] V. Putrino, D.D. Ayala, Cumulative damage assessment and strengthening efficacy of masonry buildings in Norcia affected by the 2016 seismic events in Central Italy, 2018. https://www.istructe.org/IStructE/media/Public/Resources/report-eefit-grant-cumulative-damage20181001.pdf.

[11] S. Wilkinson, D. Grant, E. Williams, S. Paganoni, S. Fraser, D. Boon, A. Mason, M. Free, Observations and implications of damage from the magnitude Mw 6.3 Christchurch, New Zealand earthquake of 22 February 2011, Bull. Earthq. Eng. 11 (2013) 107-140. https://doi.org/10.1007/s10518-012-9384-5.

[12] E. Giuriani, A. Marini, Experiences from the Northern Italy 2004 earthquake: Vulnerability assessment and strengthening of historic churches, Struct. Anal. Hist. Constr. Preserv. Saf. Significance - Proc. 6th Int. Conf. Struct. Anal. Hist. Constr. SAHC08. 1 (2008) 13-24.

[13] CEN/TC 340, EN 15129 Anti-seismic devices, 2006.

[14] R. Ceravolo, M.L. Pecorelli, L. Zanotti Fragonara, Semi-active control of the rocking motion of monolithic art objects, J. Sound Vib. 374 (2016) 1-16. https://doi.org/10.1016/j.jsv.2016.03.038.

[15] P. Clemente, A. De Stefano, R. Zago, Seismic isolation in existing complex structures, 15th World Conf. Earth. Eng., 15WCEE,(Lisbon, 24-28 Sept.), Pap. (2012). 
[16] P. Clemente, A. De Stefano, Application of seismic isolation in the retrofit of historical buildings, WIT Trans. Built Environ. 120 (2011) 41-52. https://doi.org/10.2495/ERES110041.

[17] J. Johnson, Modern Solutions to Historic Problems: The Utah state Capitol Building Seismic Retrofit Poject, Utah Preserv. (2005).

[18] A. De Luca, E. Mele, J. Molina, G. Verzeletti, A. V. Pinto, Base isolation for retrofitting historic buildings: Evaluation of seismic performance through experimental investigation, Earthq. Eng. Struct. Dyn. 30 (2001) 1125-1145. https://doi.org/10.1002/eqe.54.

[19] A.B. Habieb, M. Valente, G. Milani, Hybrid seismic base isolation of a historical masonry church using unbonded fiber reinforced elastomeric isolators and shape memory alloy wires, Eng. Struct. 196 (2019) 109281. https://doi.org/10.1016/j.engstruct.2019.109281.

[20] F.M. Mazzolani, Passive control technologies for seismic-resistant buildings in Europe, Prog. Struct. Eng. Mater. 3 (2001) 277-287. https://doi.org/10.1002/pse.83.

[21] K. Doherty, M.C. Griffith, N. Lam, J. Wilson, Displacement-based seismic analysis for out-of-plane bending of unreinforced masonry walls, Earthq. Eng. Struct. Dyn. 31 (2002) 833-850. https://doi.org/10.1002/eqe.126.

[22] M. Indirli, M.G. Castellano, Shape memory alloy devices for the structural improvement of masonry heritage structures, Int. J. Archit. Herit. 2 (2008) 93-119. https://doi.org/10.1080/15583050701636258.

[23] S. Paganoni, Dissipative Anchor Devices for the Seismic Retrofit of Heritage Buildings, Ph.D. Thesis, Univ. Bath, Dep. Archit. Civ. Eng. 1 (2015).

[24] M. Dolce, F.C. Ponzo, M. Di Croce, C. Moroni, F. Giordano, D. Nigro, R. Marnetto, F.M. Mazzolani, Experimental assessment of the CAM and DIS-CAM systems for the seismic upgrading of monumental masonry buildings, Int. Conf. Prot. Hist. Build. (2009) 1021-1027.

[25] C.E. Grigorian, T.S. Yang, E.P. Popoc, Slotted Bolted Connection Energy Dissipators, Earthq. Spectra. 9 (1993) 491-504.

[26] A. Pall, R.T. Pall, Performance-based design using Pall friction dampers - An economical design solution., 13th World Conf. Earthq. Eng. Vancouver. (2004).

[27] H.H. Khoo, G.C. Clifton, Development of the low damage self-centering Sliding Hinge Joint, Proc. Ninth Pacific Conf. Earthq. Eng. Build. an Earthquake-Resilient Soc. (2011) 1-8.

[28] M.C. Constantinou, T.T. Soong, G.F. Dargush, Passive Energy Dissipation Systems for Structural Design and Retrofit, 1998.

[29] Yuri Totoev, Design Procedure for Semi Interlocking Masonry, J. Civ. Eng. Archit. 9 (2015) 517-525. https://doi.org/10.17265/1934-7359/2015.05.003.

[30] M. Preti, S. Loda, V. Bolis, S. Cominelli, A. Marini, E. Giuriani, Dissipative Roof Diaphragm for the Seismic Retrofit of Listed Masonry Churches, J. Earthq. Eng. 23 (2019) 1241-1261. https://doi.org/10.1080/13632469.2017.1360223.

[31] V. Bolis, M. Preti, A. Marini, E. Giuriani, Experimental cyclic and dynamic in-plane rocking response of a masonry transverse arch typical of historical churches, Eng. Struct. 147 (2017) 285-296. https://doi.org/10.1016/j.engstruct.2017.05.058.

[32] O. Al Shawa, G. Felice, A. Mauro, L. Sorrentino, Out-of-plane seismic behaviour of rocking masonry walls, Earthq. Eng. Struct. Dyn. 41 (2012) 949-968. https://doi.org/10.1002/eqe.1168.

[33] P. James, D. Lee, S. Paganoni, D. D'ayala, Improvements In and Relating To Building Anchor Systems, 2011.

[34] D.F. D’Ayala, S. Paganoni, Testing and design protocol of dissipative devices for out-of-plane damage, Proc. Inst. Civ. Eng. Struct. Build. 167 (2014) 26-40. https://doi.org/10.1680/stbu.12.00087.

[35] S.C. Lim, M.F. Ashby, Wear-mechanism maps, Acta Metall. 35 (1987) 1-24.

[36] M. Latour, V. Piluso, G. Rizzano, Experimental analysis on friction materials for supplemental damping devices, Constr. Build. Mater. 65 (2014) 159-176. https://doi.org/10.1016/j.conbuildmat.2014.04.092.

[37] C. Heistermann, Resistance of Friction Connections with Open Slotted Holes in Towers for Wind Turbines, 2014. http://urn.kb.se/resolve?urn=urn:nbn:se:ltu:diva-18533.

[38] M. D'Antimo, M. Latour, G.F. Cavallaro, J.P. Jaspart, S. Ramhormozian, J.F. Demonceau, Short- and long- term loss of preloading in slotted bolted connections, J. Constr. Steel Res. 167 (2020) 105956. https://doi.org/10.1016/j.jcsr.2020.105956.

[39] V. Kodur, S. Kand, W. Khaliq, Effect of Temperature on Thermal and Mechanical Properties of Steel Bolts, J. Mater. Civ. Eng. 24 (2012) 765-774. https://doi.org/10.1061/(ASCE)MT.1943-5533.0000445.

[40] C.H. Lee, J. Ryu, J. Oh, C.H. Yoo, Y.K. Ju, Friction between a new low-steel composite material and milled steel for SAFE Dampers, Eng. Struct. 122 (2016) 279-295. https://doi.org/10.1016/j.engstruct.2016.04.056.

[41] The European Stainless Steel Development Association, Stainless Steel in Contact with Other Metallic Materials, Mater. Appl. Ser. 10 (2009) 1-24.

[42] D. D'Ayala, Y.D. Aktas, Moisture dynamics in the masonry fabric of historic buildings subjected to wind-driven rain and flooding, Build. Environ. 104 (2016) 208-220. https://doi.org/10.1016/j.buildenv.2016.05.015.

[43] Y.D. Aktas, D. D'Ayala, N. Blades, C. Calnan, An assessment of moisture induced damage in Blickling Hall in Norfolk, England, via environmental monitoring, Herit. Sci. 5 (2017). https://doi.org/10.1186/s40494-017-0119- 
4.

[44] R. Green-Warren, An Experimental Investigation of Torque Loss in a Die Cast Aluminum Threaded Fastener Joint, McNair Sch. Res. J. 11 (2018) 6.

[45] H. Mahmoud, S. Rodriguez Lopez, G. A. Riveros, Causes of Pretension Loss in High-Strength Bolts, U.S. Army Eng. Res. Dev. Cent. (2016). https://doi.org/10.13140/RG.2.2.21852.80000.

[46] S. Saha, S. Srimani, S. Hajra, A. Bhattacharya, S. Das, On the Anti-Loosening Property of Different Fasteners, in: 13th Natl. Conf. Mech. Mach. (NaCoMM07), IISc, Bangalore, India, December 12-13, 2007, 2007: pp. 229 232.

[47] A. Söderlund, Influence of Surface Flatness on Bolted Flanges, Karlstad University, 2017.

[48] EN 1090-2:2008, Execution of steel structures and aluminium structures, 2008.

[49] Rockside Export, Properties of grade 8.8 bolt \& nut (iso), (2019) 898.

[50] G. Ferrante Cavallaro, M. Latour, A.B. Francavilla, V. Piluso, G. Rizzano, Standardised friction damper bolt assemblies time-related relaxation and installed tension variability, J. Constr. Steel Res. 141 (2018) 145-155. https://doi.org/10.1016/j.jcsr.2017.10.029.

[51] Dassault Systems, Abaqus Cae, v.19, (2019).

[52] N. Nabid, I. Hajirasouliha, M. Petkovski, A practical method for optimum seismic design of friction wall dampers, Earthq. Spectra. 33 (2017) 1033-1052. https://doi.org/10.1193/110316EQS190M.

[53] M.C. Constantinou, A.M. Reinhorn, R. Watson, Teflon bearings in aseismic base isolation: experimental studies, Am. Soc. Mech. Eng. Press. Vessel. Pip. Div. PVP. 147 (1988) 9-13.

[54] C.G. Cho, M. Kwon, Development and modeling of a frictional wall damper and its applications in reinforced concrete frame structures, Earthq. Eng. Struct. Dyn. 33 (2004) 821-838. https://doi.org/10.1002/eqe.379.

[55] B.A. Mokha, M. Constantinou, A. Member, A. Reinhorn, Teflon bearings in base isolation. I: testing, J. Struct. Eng. 116 (1990) 438-454.

[56] M. Constantinou, A. Mokha, A. Reinhorn, Teflon bearings in base isolation. II: modeling, J. Struct. Eng. 116 (1990) 455-474.

[57] D. Giuliani, F. Antenucci, Valori medi climatici dal 1951 al 2000 nella regione Abruzzo, 2017.

[58] BS EN 1998:2004, Design of structures for earthquake resistance, London, 2004. 